\title{
Stochastic optimization for real time service capacity allocation under random service demand
}

\author{
Christopher Dance • Alexei A. Gaivoronski
}

Published online: 10 February 2011

(C) The Author(s) 2011. This article is published with open access at Springerlink.com

\begin{abstract}
The problem of repeated allocation of limited renewable service resources to distributed service centers is considered here. The objective is to assure a given Quality of Service expressed through percentage of demand which is satisfied during a specified time period. Resource requirements are not fully known at the time when a decision about the service resource distribution is taken.

The problem is addressed by formulating a succession of stochastic optimization problems solved at the time of resource allocation. Solutions of these problems are derived by applying duality theory. We pay special attention to the interplay between performance and risk by introducing the concept of a risk budget. Results of numerical experiments confirm the efficiency of the approach.
\end{abstract}

Keywords Stochastic optimization $\cdot$ Real time algorithms $\cdot$ Resource allocation $\cdot$ Risk budgeting

\section{Introduction}

This paper is dedicated to the development of solution procedures for repeated allocation of renewable resources in industrial environments under conditions of uncertainty. Such problems are very common in different branches of manufacturing and service industries like telecommunications, transportation, production and maintenance planning. In order to be specific we shall refer in this paper to allocation of service resources between different service sites which generate random service demand, but our approach and algorithms are applicable to a considerably wider set of problems.

C. Dance

Xerox Research Center Europe, Grenoble, France

e-mail: Chris.Dance@xrce.xerox.com

A.A. Gaivoronski ( $\bowtie)$

Norwegian University of Science and Technology, Trondheim, Norway

e-mail: Alexei.Gaivoronski@iot.ntnu.no 
Our reference setup is the following. Let us consider an enterprise that manufactures complex equipment that is sold to geographically distributed customers. This equipment requires repair and maintenance. While some part of this maintenance can be done on a periodic and predictable basis, another substantial part is generated randomly and requires quick intervention of service engineers. Usually, this maintenance is done under service agreements which specify the time constraints on repairs. Violation of these constraints can lead to substantial penalties and even to the loss of a service contract.

In order to fulfill its obligations according to the service agreements, the manufacturer sets up call centers which cater to the customers in a given geographical area. At the beginning of each decision period (usually one day) a call center allocates the available service resources measured in working time of its service engineers between different service sites following service requests. One substantial difficulty here is that the scope of required repairs and, consequently, related resource requirements will be fully known only after the arrival of a service engineer at the service site. Therefore they are known only partially when the decision is made about resource allocation. This uncertainty is modelled by considering the service demand to be random and distributed according to a known demand distribution. This resource allocation process is repeated on a daily basis as old repair requests are being satisfied and new requests arrive. The objective of resource allocation is to assure the Quality of Service (QoS) set by service contracts under constraints on available service resources. QoS requirements are formulated in terms of a (high) percentage of service requests to be satisfied within an agreed time limit. The compliance to the contract terms is subject to periodical reviewing. If QoS at review time falls short of the agreed target then substantial penalties may follow.

One can think about several ways of addressing this problem. The feature to keep in mind is that there are many decision periods in this setting. One possibility is to apply stochastic dynamic programming on a large time horizon (Bertsekas 2007). This is a theoretically optimal approach, but it faces an explosion of dimensionality as the number of demand locations increases. Despite some promising advances like stochastic dual dynamic programming (Pereira and Pinto 1991; Philpott and Guan 2008) and different approximate dynamic programming algorithms (Konda and Tsitsiklis 2003; de Farias and Van Roy 2006; Powell 2009) it still faces considerable difficulties when the number of states is large, especially in the stochastic case. Alternatively, one can employ multiperiod stochastic programming models (Birge and Louveaux 1997; Kall and Wallace 1994, see also Ruszczynski and Shapiro 2003). In this case one should approximate the demand distribution by a finite number of scenarios and construct a scenario tree. The size of this tree grows exponentially with the number of time periods, and also with the number of demand sites in the most frequent case of sites with independent demands. Thus, both these approaches can face substantial numerical difficulties when applied to problems of realistic size. An interesting alternative is to combine simulation and optimization (Pflug 1996, see also Gaivoronski 2005). In this case it is necessary to select a parametric class of decision rules and optimize the performance of the system by repeatedly simulating the system behavior which also can be time consuming.

This paper shares its general approach with work on online optimization and online stochastic optimization, see for example Van Hentenryck et al. (2009), Auer et al. (2002), Kalai and Vempala (2005), Megow and Schulz (2004). This approach has originated at the intersection of computer science, statistics and operations research and considers situations where a sequence of decisions should be made frequently in response to observations of the environment that are generated according to unknown and possibly random mechanism that is being learned in the course of optimization. The cost of decision is revealed after 
it is made and the emphasis is on the integration of learning and lightweight optimization procedures. We incorporate the learning of demand into some of our algorithms (for example, in Sect. 4), but we do not utilize the notion of regret often used in online optimization. Interesting analysis of some applied aspects of such real-time optimization models for the case of the motor carrier industry is presented in Powell et al. (2002).

The optimization problems that we consider in this paper belong to the class of problems that involve the minimization of a separable function under one separable (in our case linear) constraint. Such problems often arise in the context of resource allocation. In Patriksson (2008) one can find a detailed survey of different deterministic applications and algorithms. In the stochastic case, which we are concerned with in this paper, the closest line of research is represented by the newsvendor problem and, more specifically, by the multi-product newsvendor problem with a budget constraint. The first result in this direction dates back to Hadley and Whitin (1963) and there is substantial recent research activity, see Lau and Lau (1996), Erlebacher (2000), Abdel-Malek and Montanari (2005), Chung et al. (2008), Zhang et al. (2009), Zhang and Du (2010), where one can find additional references. One of the main tools that is used for analysis of such problems is the duality theory that allows to express the solution as a function of a single Lagrangian multiplier that corresponds to the budget constraint. We also employ the duality theory for this purpose. The closest result to this line of research that we report here is our simplest Corollary 3, which is still not deducible from the results on newsvendor problem due to the different form of the objective function.

Another research field that is relevant to our research reported in this paper is the call center modeling, dimensioning and resource allocation. This is a popular and growing research field that utilizes the methods of queueing theory, simulation and optimization, see Aksin et al. (2007) for a recent survey. Different service level agreements considered also in this paper were analyzed from the point of view of queueing theory in Baron and Milner (2009) and Milner and Olsen (2008). A stochastic programming model for call center dimensioning and resource allocation with finite number of demand scenarios is presented in Robbins and Harrison (2010). Fluid approximation of queueing systems combined with the stochastic problem with recourse for the dimensioning and resource allocation was considered by Harrison and Zeevi (2005). Different optimization models for resource allocation in call centers with several demand streams are presented in Atlason et al. (2008), Avramidis et al. (2010).

In this paper we distinguish ourselves from the previous research by developing a family of lightweight stochastic optimization models for service resource allocation, which are applied every time period for making the resource allocation decision in a moving window fashion. The purpose is to strike a balance between modeling accuracy and computational feasibility, making it possible to obtain solutions in real time even for thousands of sites. The structure of these models allows us to characterize their solutions using duality theory. These characterizations facilitate the computation, but also open the possibility for further improvement of the system performance by tuning their parameters by additional simulation and optimization. We also address the issue what to do in the cases when the distribution of demand is partially or fully unknown and/or is subject to change. This is done by integration of the ideas of adaptation and machine learning, as presented in Cesa-Bianchi and Lugosi (2006), of an appropriately defined optimal policy by incorporating newly arrived demand observations. Here we have applied learning ideas previously explored in the financial applications where certain optimality properties of similar policies were established, see Cover (1991), Gaivoronski and Stella (2003). Still another novel contribution consists in explicit modelling of the appropriate notion of risk, introducing the concept of a risk budget 
and choosing policies, which take into account the current risk budget. Summarizing, we have developed a set of fast algorithms which can quickly produce aggregated distribution of service resources between sites. This aggregated decision will serve as an input for more detailed scheduling aimed at the best utilization of these resources for performing different jobs using the methods of scheduling theory, see Dempster et al. (1982).

The rest of the paper is organized as follows. Section 2 contains a more formal description of the resource allocation process outlined above. Several solutions of related stochastic optimization problems are described in Sect. 3. The main emphasis there is to utilize duality theory in order to obtain relatively easily computable explicit characterization of decision policies. The approach of this section is further developed in different directions in Sects. 4 and 5. Section 4 presents a procedure that integrates the learning of the demand properties with resource allocation. In addition, it shows how to tune the parameters of our algorithms using simulation and optimization and starts to consider the balance between performance and risk. The risk theme is taken up also in Sect. 5. A somewhat different and more realistic problem setup is presented there, with the risks of not meeting the contract requirements on QoS being taken into consideration explicitly. The useful perspective here is the concept of risk budgeting which originated in finance and is developed here for the case of resource allocation under constraints on QoS. Also in this case duality theory allows us to derive an explicit characterization of the decision policy. Finally, Sect. 6 presents an extensive set of numerical experiments, which confirm high numerical efficiency of our approach and illustrate the performance of decision policies derived in the previous sections, providing useful insights into their properties.

\section{Problem statement}

The process evolves in discrete time $t=1, \ldots, T, \ldots$ The system under consideration consists of several sites indexed by $i=1, \ldots, m$. At each time period each of the sites generates a service demand. At the beginning of each period it is necessary to make a decision about the distribution of service capacity among sites such that some overall criterion on the Quality of Service $(\mathrm{QoS})$ will be satisfied. In what follows different elements of the problem statement are described in more detail.

Demand generation In time period $t$, each of the sites $i$ generates service demand $d_{i}^{t}$. This demand is measured in terms of the service resources necessary for its servicing. It is an observation of the random variable $d_{i}$ with distribution $H_{i}(d)$ defined on an appropriate probability space. Each site may have some local capability to meet the service demand, but we do not model this capability explicitly, denoting by $d_{i}^{t}$ demand which requires external servicing.

Demand servicing At the beginning of time period $t$ a decision should be made about the distribution of limited service resources. It is assumed that there is a finite capacity $\bar{V}$ for demand servicing. In this case the servicing decision can be expressed in terms of the servicing resource (usually time) $V_{i}^{t}$ allocated to site $i$ during time period $t$. Then we have the following constraint on the distribution of service resources:

$$
\sum_{i=1}^{m} V_{i}^{t} \leq \bar{V}
$$


The decision is taken without full knowledge of actual service demand and on the basis of distributions $H_{i}(d)$.

Alternatively, the service decision can consist in defining where to send service personnel during this period. Then the decision is described by binary variables $u_{i}^{t}$ which equal one if site $i$ is serviced during period $t$ and zero otherwise. The maximal amount of sites under service during each given period is $U$ :

$$
\sum_{i=1}^{m} u_{i}^{t} \leq U
$$

where $U \leq m$. The binary nature of control variables $u_{i}^{t}$ corresponds to the decisions to send one or zero persons to the site. More generally, one can assume that $u_{i}^{t}$ takes integer or even real values within some bounds:

$$
0 \leq u_{i}^{t} \leq U_{i}
$$

The real numbers here take into account that the process repeats many times and refer to the average number of persons to send to a given location.

Decisions taken in terms of service resources (1) can be transformed in decisions in terms of sent personnel (2) by making assumptions about the quantity of service resource $V_{0}$ which one person provides. This transformation will result in fractions of persons which can be interpreted as the average number of persons to send, given that the process repeats many times. This will give an approximate solution, while more precise solution of the problem with discrete resources requires different techniques and is not considered here. In the rest of the paper we shall consider demand servicing in terms of allocation of service resources (1).

It is necessary to specify what happens with unserved demand. Some alternatives:

- Backlogs are not allowed and not served demand during a given time period is lost. For example, it can be satisfied by some emergency structure.

- Backlogs are allowed. Then unserved demand from period $t$ can be served in the next period or even later (with an appropriate penalty). In this case we have the following equation for accumulated demand $w_{i}^{t}$ at site $i$ to serve during time period $t$ expressed in terms of required service resource:

$$
w_{i}^{t+1}=d_{i}^{t+1}+\max \left\{0, w_{i}^{t}-V_{i}^{t}\right\}
$$

Performance measures It is assumed that served demand brings benefit while unserved demand brings penalties. Originally, performance is expressed on a per customer basis in terms of Quality of Service (QoS) which in its turn is defined as the percentage of jobs serviced during a given time period. Penalties are paid if given QoS objectives are not met. In this paper we work with the aggregated demand description and therefore the notion of QoS is approximated by the fraction of resource demand which is met. This leads to performance measures expressed in terms of benefit for satisfied resource demand and penalties for nonsatisfied one.

Let us introduce the following notations:

$a_{i}$-profit which comes from servicing a unit of demand at location $i$ during one time period;

$b_{i}$ - cost which comes from not serving a unit of demand at location $i$ during one time period; 
Some of our subsequent results can be extended to the case when $a_{i}$ and $b_{i}$ depend on time, but we shall not pursue this issue further.

Thus, the contribution of site $i$ at time $t$ to the overall performance is

$$
f_{i}^{t}\left(V_{i}^{t}\right)=a_{i} \min \left\{w_{i}^{t}, V_{i}^{t}\right\}-b_{i} \max \left\{0, w_{i}^{t}-V_{i}^{t}\right\}
$$

where we have not indicated explicitly the dependence of $f_{i}^{t}\left(V_{i}^{t}\right)$ on $w_{i}^{t}$ in order to simplify notations. This expression is valid for the case when backlogs are allowed as in (3). In the case when backlogs are not allowed one can use (4) after substituting $w_{i}^{t}$ for $d_{i}^{t}$. The total performance of the system at time $t$ is

$$
F^{t}\left(V^{t}\right)=\sum_{i=1}^{m} \lambda_{i} f_{i}^{t}\left(V_{i}^{t}\right)
$$

where $\lambda_{i}$ are non-negative weight coefficients reflecting the different importance of different sites. The overall measure of performance over $T$ time periods is

$$
F(V(T))=\frac{1}{T} \sum_{t=1}^{T} F^{t}\left(V^{t}\right)
$$

where $V^{t}=\left(V_{1}^{t}, \ldots, V_{m}^{t}\right), V(T)=\left(V^{1}, \ldots, V^{T}\right)$. On the infinite time horizon this will assume the form

$$
F\left(V_{\infty}\right)=\lim _{T \rightarrow \infty} \frac{1}{T} \sum_{t=1}^{T} F^{t}\left(V^{t}\right)
$$

where $V_{\infty}$ is defined similarly to $V(T)$ but contains infinite sequences. Another component of the costs can come from reassignment. The reassignment costs at time $t$ will be

$$
c_{t}\left(V^{t}, V^{t-1}\right)=\sum_{i=1}^{m} \delta_{i}\left\|V_{i}^{t}-V_{i}^{t-1}\right\|
$$

where $\|\cdot\|$ is an appropriate measure of distance between different resource assignments that can approximate reassignment of personnel but also the different nature of jobs that require different expenditure of resources. The reassignment costs $\delta_{i}$ may be site specific and reflect the technological diversity of different sites and other characteristics like geographical location. In this case the total performance of the system at time $t$ will be

$$
F^{t}\left(V^{t}, V^{t-1}\right)=\sum_{i=1}^{m} \lambda_{i} f_{i}^{t}\left(V_{i}^{t}\right)-c_{t}\left(V^{t}, V^{t-1}\right)
$$

\section{Solution approaches}

In this section we propose different solution techniques for the service model described in the previous section. Summarizing our discussion so far, the problem to solve will be

$$
\max _{V(T) \geq 0} \mathbb{E} \sum_{t=1}^{T}\left(\sum_{i=1}^{m} \lambda_{i} f_{i}^{t}\left(V_{i}^{t}\right)-c_{t}\left(V^{t}, V^{t-1}\right)\right)
$$


subject to constraints

$$
\sum_{i=1}^{m} V_{i}^{t} \leq \bar{V}
$$

where $f_{i}^{t}\left(V_{i}^{t}\right)$ is defined in (4) and expectation is taken with respect to random demands. Component $V^{t}$ of this solution will be a function of $V^{1}, \ldots, V^{t-1}$ and all demand observations $d^{1}, \ldots, d^{t-1}$ prior to time period $t$, where $d^{t}=\left(d_{1}^{t}, \ldots, d_{m}^{t}\right)$. Exact solution of this problem requires application of stochastic dynamic programming which will be unfeasible for realistic values of $T$ and $m$. Therefore different computationally feasible approaches should be developed either by simplifying the problem formulation or by reducing the set of possible strategies from which the solution is selected. In the rest of this section several such solutions on reduced strategy sets will be developed.

\subsection{Stationary solution}

Suppose that no backlogs are allowed and demand at time period $t+1$ is independent from demand at time period $t$. Besides, let us assume that the demand distribution does not change with time. In this case the objective function (6) decomposes with respect to the time axis and a solution will be found in the set of constant strategies, such that the switching costs will vanish. Since in this case the random variables $d_{i}^{t}$ are identically distributed for all $t$ (but not necessarily for all $i$ ) we obtain that due to the law of large numbers

$$
F\left(V_{\infty}\right)=\mathbb{E} F^{t}\left(V^{t}\right)
$$

where $t$ is arbitrary and the expectation is taken with respect to random variables $d_{i}^{t}$. This together with (4) and (5) will yield the following optimization problem for finding $V_{i}^{t}$, where we have dropped the dependence on $t$ because the solution will not depend on time:

$$
\begin{aligned}
& \max _{V_{i} \geq 0} \mathbb{E} \sum_{i=1}^{m} \lambda_{i}\left(a_{i} \min \left\{d_{i}, V_{i}\right\}-b_{i} \max \left\{0, d_{i}-V_{i}\right\}\right) \\
& \sum_{i=1}^{m} V_{i} \leq \bar{V}
\end{aligned}
$$

We shall obtain the explicit solution of this problem as a corollary of the solution of a more general problem that we describe below (see Corollary 3 below).

Let us look now at the case when at the beginning of some time period it is necessary to obtain the current service allocation taking into account reallocation costs from the allocation $V^{0}$ utilized during the previous time period. Such a necessity can arise when the demand distribution was updated due to processing of new observations. Then it is necessary to solve the following one period problem:

$$
\max _{V_{i} \geq 0} \mathbb{E} \sum_{i=1}^{m} \lambda_{i}\left(a_{i} \min \left\{d_{i}, V_{i}\right\}-b_{i} \max \left\{0, d_{i}-V_{i}\right\}\right)-\sum_{i=1}^{m} \delta_{i}\left|V_{i}-V_{i}^{0}\right|
$$

under constraint (13). We have obtained (14) from (8), (9), taking the absolute value as the norm in (8). The problem is concave and the duality theory can be employed in order to characterize its solution. 
Proposition 1 Suppose that the joint distribution of demand has bounded marginal densities $h_{i}(y), i=1: m$ and $\sup _{y}\left\{y \mid h_{i}(y)>0\right\}=d_{i}^{+}<\infty$.

Then the solution $V^{*}=\left(V_{1}^{*}, \ldots, V_{m}^{*}\right)$ of problem (14), (13) exists and has the following form:

$$
V_{i}^{*}= \begin{cases}\hat{V}_{i} & \text { if } \sum_{i=1}^{m} \hat{V}_{i} \leq \bar{V} \\ V_{i}\left(\mu^{*}\right) & \text { otherwise }\end{cases}
$$

where

$$
\hat{V}_{i}= \begin{cases}V_{i}^{0} & \text { if } \lambda_{i}\left(a_{i}+b_{i}\right) \mathbb{P}\left\{d_{i} \geq V_{i}^{0}\right\}<\delta_{i}, \delta_{i}>0 \\ \inf _{y}\left\{y \mid \lambda_{i}\left(a_{i}+b_{i}\right) \mathbb{P}\left\{d_{i} \geq y\right\}=\delta_{i}\right\} & \text { if } \lambda_{i}\left(a_{i}+b_{i}\right) \mathbb{P}\left\{d_{i} \geq V_{i}^{0}\right\} \geq \delta_{i}, \delta_{i}>0 \\ d_{i}^{+} & \text {if } \delta_{i}=0\end{cases}
$$

and $\mu^{*}$ satisfies equation

$$
\sum_{i=1}^{m} V_{i}(\mu)=\bar{V}
$$

where $V_{i}(\mu)$ is a selection from the mapping

$$
V_{i}(\mu) \in \begin{cases}0 & \text { if } \mu>\lambda_{i}\left(a_{i}+b_{i}\right)+\delta_{i} \\ {\left[0, d_{i}^{-}\right]} & \text {if } \mu=\lambda_{i}\left(a_{i}+b_{i}\right)+\delta_{i} \\ {\left[V_{i}^{-}\left(\delta_{i}, \mu\right), V_{i}^{+}\left(\delta_{i}, \mu\right)\right]} & \text { if } \lambda_{i}\left(a_{i}+b_{i}\right) \mathbb{P}\left\{d_{i} \geq V_{i}^{0}\right\}+\delta_{i} \leq \mu<\lambda_{i}\left(a_{i}+b_{i}\right)+\delta_{i} \\ V_{i}^{0} & \text { if } \lambda_{i}\left(a_{i}+b_{i}\right) \mathbb{P}\left\{d_{i} \geq V_{i}^{0}\right\}-\delta_{i} \\ & \quad<\mu<\lambda_{i}\left(a_{i}+b_{i}\right) \mathbb{P}\left\{d_{i} \geq V_{i}^{0}\right\}+\delta_{i} \\ & \quad \text { if } 0 \leq \mu \leq \lambda_{i}\left(a_{i}+b_{i}\right) \mathbb{P}\left\{d_{i} \geq V_{i}^{0}\right\}-\delta_{i}\end{cases}
$$

with $d_{i}^{-}=\inf _{y}\left\{y \mid h_{i}(y)>0\right\}$ and

$$
\begin{aligned}
& V_{i}^{-}\left(\delta_{i}, \mu\right)=\inf _{y}\left\{y \mid \mu=\lambda_{i}\left(a_{i}+b_{i}\right) \mathbb{P}\left\{d_{i} \geq y\right\}+\delta_{i}\right\} \\
& V_{i}^{+}\left(\delta_{i}, \mu\right)=\sup _{y}\left\{y \mid \mu=\lambda_{i}\left(a_{i}+b_{i}\right) \mathbb{P}\left\{d_{i} \geq y\right\}+\delta_{i}\right\} \\
& \hat{V}_{i}^{-}\left(\delta_{i}, \mu\right)=\inf _{y}\left\{y \mid \mu=\lambda_{i}\left(a_{i}+b_{i}\right) \mathbb{P}\left\{d_{i} \geq y\right\}-\delta_{i}\right\} \\
& \hat{V}_{i}^{+}\left(\delta_{i}, \mu\right)=\sup _{y}\left\{y \mid \mu=\lambda_{i}\left(a_{i}+b_{i}\right) \mathbb{P}\left\{d_{i} \geq y\right\}-\delta_{i}\right\}
\end{aligned}
$$

Proof Let us denote

$$
\begin{aligned}
& \varphi_{i}\left(V_{i}, d_{i}\right)=\lambda_{i}\left(a_{i} \min \left\{d_{i}, V_{i}\right\}-b_{i} \max \left\{0, d_{i}-V_{i}\right\}\right)-\delta_{i}\left|V_{i}-V_{i}^{0}\right| \\
& \Phi(V, d)=\sum_{i=1}^{m} \varphi_{i}\left(V_{i}, d_{i}\right)
\end{aligned}
$$

Observe that $\mathbb{E} \Phi(V, d)$ is the objective function in (14). 
1. Since

$$
\begin{aligned}
& \left|\min \left\{d_{i}, V_{i}^{1}\right\}-\min \left\{d_{i}, V_{i}^{2}\right\}\right| \leq\left|V_{i}^{1}-V_{i}^{2}\right| \\
& \left|\max \left\{0, d_{i}-V_{i}^{1}\right\}-\max \left\{0, d_{i}-V_{i}^{2}\right\}\right| \leq\left|V_{i}^{1}-V_{i}^{2}\right| \\
& || V_{i}^{1}-V_{i}^{0}|-| V_{i}^{2}-V_{i}^{0}|| \leq\left|V_{i}^{1}-V_{i}^{2}\right|
\end{aligned}
$$

we have the following inequality for arbitrary $V^{k}=\left(V_{1}^{k}, \ldots, V_{m}^{k}\right), k=1,2$ :

$$
\begin{aligned}
\left|\mathbb{E} \Phi\left(V^{1}, d\right)-\mathbb{E} \Phi\left(V^{2}, d\right)\right| & \leq \mathbb{E}\left|\Phi\left(V^{1}, d\right)-\Phi\left(V^{2}, d\right)\right| \leq \mathbb{E} \sum_{i=1}^{m}\left|\varphi_{i}\left(V_{i}^{1}, d_{i}\right)-\varphi_{i}\left(V_{i}^{2}, d_{i}\right)\right| \\
& \leq \sum_{i=1}^{m}\left(\lambda_{i} a_{i}+\lambda_{i} b_{i}+\delta_{i}\right)\left|V_{i}^{1}-V_{i}^{2}\right|
\end{aligned}
$$

which proves the continuity of the objective function in (14). This together with the compactness of the feasible set (13) proves the existence of solution of the problem (14), (13).

2. Let us find the solution of problem (14) without constraint (13). In the absence of this constraint the problem decomposes into $m$ independent problems

$$
\max _{V_{i} \geq 0} \mathbb{E} \varphi_{i}\left(V_{i}, d_{i}\right)
$$

We have:

$$
\begin{aligned}
\psi\left(V_{i}\right)= & \mathbb{E} \varphi_{i}\left(V_{i}, d_{i}\right) \\
= & \lambda_{i} a_{i} \int_{0}^{V_{i}} y h_{i}(y) d y \\
& -\lambda_{i} b_{i} \int_{V_{i}}^{\infty} y h_{i}(y) d y+\lambda_{i}\left(a_{i}+b_{i}\right) V_{i} \int_{V_{i}}^{\infty} h_{i}(y) d y-\delta_{i}\left|V_{i}-V_{i}^{0}\right|
\end{aligned}
$$

Differentiating this function with respect to $V_{i}$ yields:

$$
\frac{\partial \psi\left(V_{i}\right)}{\partial V_{i}}= \begin{cases}\lambda_{i}\left(a_{i}+b_{i}\right) \mathbb{P}\left\{d_{i} \geq V_{i}\right\}-\delta_{i} & \text { if } V_{i}>V_{i}^{0} \\ \lambda_{i}\left(a_{i}+b_{i}\right) \mathbb{P}\left\{d_{i} \geq V_{i}\right\}+\delta_{i} & \text { if } V_{i}<V_{i}^{0}\end{cases}
$$

Consequently, $\psi\left(V_{i}\right)$ will increase with increasing $V_{i}$ in the range $\left[0, V_{i}^{0}\right]$ if $\delta_{i}>0$ and if $\delta_{i}=0$ then it will increase in the range $\left[0, \min \left\{V_{i}^{0}, d_{i}^{+}\right\}\right]$. For $V_{i} \geq V_{i}^{0}$ there are two possibilities in the case $\delta_{i}>0$. If $\phi\left(V_{i}\right)=\lambda_{i}\left(a_{i}+b_{i}\right) \mathbb{P}\left\{d_{i} \geq V_{i}\right\}<\delta_{i}$ for $V_{i}=V_{i}^{0}$ then $\psi\left(V_{i}\right)$ will start decreasing immediately after $V_{i}$ becomes larger than $V_{i}^{0}$ and, consequently, the maximum will be attained at $V_{i}=V_{i}^{0}$. Otherwise this function will continue to increase until $\phi\left(V_{i}\right)$ will become equal to $\delta_{i}$ and will start to decrease when $\phi\left(V_{i}\right)$ will become less than $\delta_{i}$. If $\delta_{i}=0$ then $\psi\left(V_{i}\right)$ will continue to increase until $V_{i}$ reaches $d_{i}^{+}$and will remain constant after this. Summarizing this argument, we obtain the following description for the solution $\hat{V}=\left(\hat{V}_{1}, \ldots, \hat{V}_{m}\right)$ of problem $(19)$ :

$$
\hat{V}_{i}= \begin{cases}V_{i}^{0} & \text { if } \lambda_{i}\left(a_{i}+b_{i}\right) \mathbb{P}\left\{d_{i} \geq V_{i}^{0}\right\}<\delta_{i}, \delta_{i}>0 \\ {\left[V_{i}^{-}\left(\delta_{i}\right), V_{i}^{+}\left(\delta_{i}\right)\right]} & \text { if } \lambda_{i}\left(a_{i}+b_{i}\right) \mathbb{P}\left\{d_{i} \geq V_{i}^{0}\right\} \geq \delta_{i}, \delta_{i}>0 \\ {\left[d_{i}^{+}, \infty\right)} & \text { if } \delta_{i}=0\end{cases}
$$


where

$$
\begin{aligned}
& V_{i}^{-}\left(\delta_{i}\right)=\inf _{y}\left\{y \mid \lambda_{i}\left(a_{i}+b_{i}\right) \mathbb{P}\left\{d_{i} \geq y\right\}=\delta_{i}\right\} \\
& V_{i}^{+}\left(\delta_{i}\right)=\sup _{y}\left\{y \mid \lambda_{i}\left(a_{i}+b_{i}\right) \mathbb{P}\left\{d_{i} \geq y\right\}=\delta_{i}\right\}
\end{aligned}
$$

where both $V_{i}^{-}\left(\delta_{i}\right)$ and $V_{i}^{+}\left(\delta_{i}\right)$ exist due to the boundedness of $h_{i}(y)$. Comparing this with (16) and the first line of (15) we see that in this case there exists the solution (16) of unconstrained problems (19) that at the same time satisfies constraint (13) and, consequently, it is a solution of the original problem (14), (13). Moreover, if (16) does not satisfy (13) then (20) shows that no solution of (19) satisfies (13) and, consequently, the constraint (13) is binding and it is satisfied with equality at any solution of the problem (14), (13).

3. It remains to consider the case when the constraint (13) is binding, i.e. it is satisfied with equality. In this case we shall utilize the duality theory and exploit the equivalence of the problem (14), (13) with the problem

$$
\begin{array}{rl}
\min _{\mu \geq 0} \max _{V_{i} \geq 0} & \mathbb{E} \sum_{i=1}^{m} \lambda_{i}\left(a_{i} \min \left\{d_{i}, V_{i}\right\}-b_{i} \max \left\{0, d_{i}-V_{i}\right\}\right)-\sum_{i=1}^{m} \delta_{i}\left|V_{i}-V_{i}^{0}\right| \\
& -\mu\left(\sum_{i=1}^{m} V_{i}-\bar{V}\right)
\end{array}
$$

which we can do due to the concavity of the objective function in (14) with respect to $V$ and the linearity of constraint (13). Let us consider the inner maximization problem in (21). Similarly to (19) it decomposes into $m$ optimization problems, which this time depend on the Lagrangian multiplier $\mu$ :

$$
\begin{array}{rl}
\max _{V_{i} \geq 0} & \mathbb{E} \bar{\varphi}_{i}\left(\mu, V_{i}, d_{i}\right) \\
& \bar{\varphi}_{i}\left(\mu, V_{i}, d_{i}\right)=\lambda_{i}\left(a_{i} \min \left\{d_{i}, V_{i}\right\}-b_{i} \max \left\{0, d_{i}-V_{i}\right\}\right)-\delta_{i}\left|V_{i}-V_{i}^{0}\right|-\mu V_{i}
\end{array}
$$

where we have neglected the term $\mu \bar{V}$ that does not depend on $V_{i}$. Expressing the expectations through integrals we obtain for $\bar{\psi}\left(\mu, V_{i}\right)=\mathbb{E} \bar{\varphi}_{i}\left(\mu, V_{i}, d_{i}\right)$ :

$$
\begin{aligned}
\bar{\psi}\left(\mu, V_{i}\right)= & \lambda_{i} a_{i} \int_{0}^{V_{i}} y h_{i}(y) d y \\
& -\lambda_{i} b_{i} \int_{V_{i}}^{\infty} y h_{i}(y) d y+\lambda_{i}\left(a_{i}+b_{i}\right) V_{i} \int_{V_{i}}^{\infty} h_{i}(y) d y-\delta_{i}\left|V_{i}-V_{i}^{0}\right|-\mu V_{i} .
\end{aligned}
$$

Differentiating this with respect to $V_{i}$ yields:

$$
\frac{\partial \bar{\psi}\left(\mu, V_{i}\right)}{\partial V_{i}}= \begin{cases}\lambda_{i}\left(a_{i}+b_{i}\right) \mathbb{P}\left\{d_{i} \geq V_{i}\right\}-\mu-\delta_{i} & \text { if } V_{i}>V_{i}^{0} \\ \lambda_{i}\left(a_{i}+b_{i}\right) \mathbb{P}\left\{d_{i} \geq V_{i}\right\}-\mu+\delta_{i} & \text { if } V_{i}<V_{i}^{0}\end{cases}
$$

Investigation of the sign of this derivative yields the following observations. The function $\bar{\psi}\left(\mu, V_{i}\right)$ will decrease with increasing $V_{i}$ in the whole domain $V_{i} \geq 0$ if $\mu>\lambda_{i}\left(a_{i}+b_{i}\right)+\delta_{i}$, consequently it attains its maximum at $V_{i}=0$. In the case when $\mu=\lambda_{i}\left(a_{i}+b_{i}\right)+\delta_{i}$ this 
function will remain at its maximum in the range $\left[0, d_{i}^{-}\right]$and then starts to decrease, this is due to the boundedness of $h_{i}(y)$. If $\mu<\lambda_{i}\left(a_{i}+b_{i}\right)+\delta_{i}$, but $\mu \geq \lambda_{i}\left(a_{i}+b_{i}\right) \mathbb{P}\left\{d_{i} \geq\right.$ $\left.V_{i}^{0}\right\}+\delta_{i}$ then the function $\bar{\psi}\left(\mu, V_{i}\right)$ will be increasing on the range $\left[0, V_{i}^{-}\left(\delta_{i}, \mu\right)\right]$ with $V_{i}^{-}\left(\delta_{i}, \mu\right) \leq V_{i}^{0}$, constant on the range $\left[V_{i}^{-}\left(\delta_{i}, \mu\right), V_{i}^{+}\left(\delta_{i}, \mu\right)\right]$ and decreasing on the range $\left[V_{i}^{-}\left(\delta_{i}, \mu\right), \infty\right]$. If $\mu<\lambda_{i}\left(a_{i}+b_{i}\right) \mathbb{P}\left\{d_{i} \geq V_{i}^{0}\right\}+\delta_{i}$, but $\mu>\lambda_{i}\left(a_{i}+b_{i}\right) \mathbb{P}\left\{d_{i} \geq V_{i}^{0}\right\}-\delta_{i}$ then this function will be increasing on the range $\left[0, V_{i}^{0}\right]$ and decreasing afterwards, attaining its maximum at $V_{i}=V_{i}^{0}$. If, instead, $\mu \leq \lambda_{i}\left(a_{i}+b_{i}\right) \mathbb{P}\left\{d_{i} \geq V_{i}^{0}\right\}-\delta_{i}$ then the function $\bar{\psi}\left(\mu, V_{i}\right)$ will continue to grow until the point $\hat{V}_{i}^{-}\left(\delta_{i}, \mu\right)$ and start to decrease after the point $\hat{V}_{i}^{+}\left(\delta_{i}, \mu\right)$, remaining constant on the range $\left[\hat{V}_{i}^{-}\left(\delta_{i}, \mu\right), \hat{V}_{i}^{+}\left(\delta_{i}, \mu\right)\right]$. Summarizing this argument, we obtain the expression (18) for the solutions $V_{i}(\mu)$ of the problem (22). Some of the ranges in (18) can be empty, while others can extend to infinity.

We have proved already that the problem (14), (13) has solution, and according to the duality theory for any such solution there exists $\mu$ such that this solution satisfies (18). Since we are considering the case when the constraint (13) is binding this means that there exist selection $V(\mu)$ from (18) that satisfies equation (17). Conversely, any selection from (18) that satisfies (17) is a solution of (14), (13). The proof is completed.

The Proposition 1 is quite general and covers many specific cases. In particular, the representation (18) covers also the cases when a site generates a mixture of two types of demand. The first one is relatively moderate maintenance demand and the second one is much larger, but also less frequent one, which is generated by major malfunctions and/or unusually high workload. Such composite demand will be described by a distribution with disconnected support. In this case $V_{i}(\mu)$ may be nonunique as (18) shows.

Let us consider two important specific cases that are the corollaries of this proposition. They address the most practically important cases when there are no superfluous resources and all available resources are distributed between sites every time period.

Corollary 2 Suppose that the conditions of Proposition 1 are satisfied, that constraint (13) is binding and that the marginal densities $d_{i}(y)$ are bounded and have connected support that includes 0 . Then the solution of problem (14), (13) exists and has the form $V\left(\mu^{*}\right)=$ $\left(V_{1}^{*}\left(\mu^{*}\right), \ldots, V_{m}^{*}\left(\mu^{*}\right)\right)$ where $\mu^{*}$ is the solution of $(17)$ and

$$
V_{i}(\mu)=\left\{\begin{array}{lc}
0 & \text { if } \mu \geq \lambda_{i}\left(a_{i}+b_{i}\right)+\delta_{i} \\
y_{\mu}: \mathbb{P}\left\{d_{i} \geq y_{\mu}\right\}=\frac{\mu-\delta_{i}}{\lambda_{i}\left(a_{i}+b_{i}\right)} & \text { if } \lambda_{i}\left(a_{i}+b_{i}\right) \mathbb{P}\left\{d_{i} \geq V_{i}^{0}\right\}+\delta_{i} \\
& \quad \leq \mu<\lambda_{i}\left(a_{i}+b_{i}\right)+\delta_{i} \\
V_{i}^{0} & \text { if } \lambda_{i}\left(a_{i}+b_{i}\right) \mathbb{P}\left\{d_{i} \geq V_{i}^{0}\right\}-\delta_{i} \\
& \quad<\mu<\lambda_{i}\left(a_{i}+b_{i}\right) \mathbb{P}\left\{d_{i} \geq V_{i}^{0}\right\}+\delta_{i} \\
\hat{y}_{\mu}: \mathbb{P}\left\{d_{i} \geq \hat{y}_{\mu}\right\}=\frac{\mu+\delta_{i}}{\lambda_{i}\left(a_{i}+b_{i}\right)} & \text { if } 0 \leq \mu \leq \lambda_{i}\left(a_{i}+b_{i}\right) \mathbb{P}\left\{d_{i} \geq V_{i}^{0}\right\}-\delta_{i}
\end{array}\right.
$$

The representation (23) follows from (18) because under conditions of this corollary $d_{i}^{-}=0, V_{i}^{-}\left(\delta_{i}, \mu\right)=V_{i}^{+}\left(\delta_{i}, \mu\right)=y_{\mu}, \hat{V}_{i}^{-}\left(\delta_{i}, \mu\right)=\hat{V}_{i}^{+}\left(\delta_{i}, \mu\right)=\hat{y}_{\mu}$. The following corollary describes the solution of the stationary problem (12)-(13).

Corollary 3 Suppose that the conditions of Corollary 2 are satisfied and in addition the switching costs $\delta_{i}=0$. Then the solution of problem (14), (13) exists and has the form 
$V\left(\mu^{*}\right)=\left(V_{1}^{*}\left(\mu^{*}\right), \ldots, V_{m}^{*}\left(\mu^{*}\right)\right)$ where $\mu^{*}$ is the solution of $(17)$ and

$$
V_{i}(\mu)= \begin{cases}0 & \text { if } \mu \geq \lambda_{i}\left(a_{i}+b_{i}\right) \\ \hat{y}_{\mu}: \mathbb{P}\left\{d_{i} \geq \hat{y}_{\mu}\right\}=\frac{\mu}{\lambda_{i}\left(a_{i}+b_{i}\right)} & \text { otherwise }\end{cases}
$$

Conditions of this Proposition can be further relaxed. In particular, it is possible to consider the case when there are no continuous marginal densities $h_{i}\left(y_{i}\right)$ of demand, but the demand takes some discrete values with given probabilities. We shall show what kind of result can be obtained in the case of discrete distributions in Proposition 4.

Summarizing, in order to compute solution of problem (12)-(13) or (14), (13) it is necessary to solve (17) in one variable. This can be done, for example, by golden section search because the methods that use derivatives may not work due to nondifferentiability of $V_{i}(\mu)$. In the case of Corollary 3 one can take as the initial interval for this search the interval $\left[0, \max _{i} \lambda_{i}\left(a_{i}+b_{i}\right)\right]$. The search will find the solution because the sum in the left hand side of (17) monotonically change on this interval from $\sum_{i} d_{i}^{+}$to 0 . In order to compute the left hand side of this equation it is necessary to solve $m$ equations (24), which require repetitive computations of probabilities $\mathbb{P}\left(d_{i} \geq z\right)$. This is a numerically feasible problem, which reduces to one-dimensional numerical integration. Similar numerical considerations are valid also for Corollary 2 and problem (14), (13).

\section{Combining allocation decisions with the learning of demand properties}

The allocation of the service resources according to (15)-(18) assumes the knowledge of the distribution of service demand $H_{i}(y)$ and its stationarity. Both assumptions are not necessarily satisfied in practice. In such cases it is necessary to combine this allocation with learning of statistical properties of demand and/or tracking of these properties in the case of nonstationarity. One way to do this is to select a class of parametric distributions and use the previous observations of demand to estimate the parameters of these distributions. These estimates will be updated each time period and the updated distributions will be used in (15)-(18). However, this approach can introduce modeling errors, especially in the case of demand nonstationarity.

In this section we pursue the alternative approach that is often used in machine learning: select the decision to be applied at time period $t$ by optimizing the performance on some time horizon immediately preceding the current time period, like in Cover (1991), Cesa-Bianchi and Lugosi (2006), Kalai and Vempala (2005). We select the time horizon to be of length $\tau$ for which we have observations $d_{i}^{s}$ of demand for $s=t-\tau, \ldots, t-1$ and compute the fixed resource allocation which will give the average best performance for this time horizon. In this case the optimization problem that corresponds to (12) takes the form

$$
\max _{V_{i} \geq 0} \sum_{s=t-\tau}^{t-1} \gamma_{s} \sum_{i=1}^{m} \lambda_{i}\left(a_{i} \min \left\{d_{i}^{s}, V_{i}\right\}-b_{i} \max \left\{0, d_{i}^{s}-V_{i}\right\}\right)
$$

subject to constraint (13) and similarly for the case (14). The solution of this problem will be used for the service resource allocation during period $t$. By the beginning of period $t+1$ the new demand observations $d_{i}^{t}$ become known and the problem (25) will be solved again on the time window advanced by one time period and the new solution will be used during period $t+1$. The process will continue further in a similar fashion. 
This approach allows to compute the allocation decision without knowing the distribution of demand, including the cases when the distribution is nonstationary and even when the demand is generated by some nonprobabilistic mechanism. In the case when the demand is probabilistic and stationary the solution of (25) will give an approximation to the solution of the original problem obtained by using the empirical distribution. The results of the previous section are not applicable here because the basic assumption in that section was the existence and knowledge of the marginal densities $h_{i}(y)$. Thus, this section shows how to compute the allocation policy in the case of discrete demand distributions.

The nonnegative weights $\gamma_{s}$ satisfy condition

$$
\sum_{s=t-\tau}^{t-1} \gamma_{s}=1
$$

and in the simplest case can be chosen to be $\gamma_{s} \equiv 1 / \tau$. In the stationary case this will correspond to the empirical distribution. In other cases they can be chosen in such a way as to reflect trends in the demand properties with the more distant observations having less weight than the more recent ones.

We are going to derive an expression for explicit solution of problem (25) on which to base the algorithm for the service resource allocation. In order to do this let us introduce notations similar to those used for order statistics. Namely, by $d_{i}^{[s]}, s=1, \ldots, \tau$ we shall denote an arbitrary ordering of $d_{i}^{k}, k=t-\tau, \ldots, t-1$ such that

$$
d_{i}^{[s]} \leq d_{i}^{[s+1]}, \quad s=1, \ldots, \tau-1
$$

In these notations we have

$$
d_{i}^{[1]}=\min _{t-\tau \leq k \leq t-1} d_{i}^{k}, \quad d_{i}^{[\tau]}=\max _{t-\tau \leq k \leq t-1} d_{i}^{k}
$$

Proposition 4 Suppose that the constraint (13) is binding, then there exists a solution $V^{*}=$ $\left(V_{1}^{*}, \ldots, V_{m}^{*}\right)$ of problem (25) such that $V_{i}^{*} \in V_{i}\left(\mu^{*}\right)$ for all $i=1: m$ and for some $\mu^{*} \geq 0$, where $V_{i}(\mu)$ is the following set valued mapping:

$$
\begin{aligned}
& V_{i}(\mu) \\
& \quad= \begin{cases}{\left[d_{i}^{[\tau]}, \infty\right)} & \text { if } \mu=0 \\
\left\{d_{i}^{[\tau]}\right\} & \text { if } 0<\mu<\lambda_{i}\left(a_{i}+b_{i}\right) \gamma_{[\tau]} \\
\left\{d_{i}^{[k]}\right\} & \text { if } \lambda_{i}\left(a_{i}+b_{i}\right) \sum_{s=k+1}^{\tau} \gamma_{[s]}<\mu<\lambda_{i}\left(a_{i}+b_{i}\right) \sum_{s=k}^{\tau} \gamma_{[s]}, k=1: \tau-1 \\
{\left[d_{i}^{[k]}, d_{i}^{[k+1]}\right]} & \text { if } \mu=\lambda_{i}\left(a_{i}+b_{i}\right) \sum_{s=k+1}^{\tau} \gamma_{[s]}, k=1: \tau-1 \\
{\left[0, d_{i}^{[1]}\right]} & \text { if } \mu=\lambda_{i}\left(a_{i}+b_{i}\right) \\
\{0\} & \text { if } \mu>\lambda_{i}\left(a_{i}+b_{i}\right)\end{cases}
\end{aligned}
$$

Proof Existence of a solution here is guaranteed by compactness of the feasible set and continuity of the objective function. The rest of the proof is similar to the proof of Proposition 1 and is based again on duality theory. The objective function in (25) is concave and for this reason the solution of this problem is equivalent to the following dual problem:

$$
\min _{\mu \geq 0} \sum_{i=1}^{m} \max _{V_{i} \geq 0}\left(\sum_{s=t-\tau}^{t-1} \gamma_{s} \lambda_{i}\left(a_{i} \min \left\{d_{i}^{s}, V_{i}\right\}-b_{i} \max \left\{0, d_{i}^{s}-V_{i}\right\}\right)-\mu V_{i}\right)+\mu \bar{V}
$$


Thus, the inner maximization problem is decomposed into $m$ independent optimization problems which correspond to individual sites. Let us consider one such problem denoting $V_{i}$ by $z$ in order to simplify the notation:

$$
\max _{z \geq 0}\left(\sum_{s=t-\tau}^{t-1} \gamma_{s} \lambda_{i}\left(a_{i} \min \left\{d_{i}^{s}, z\right\}-b_{i} \max \left\{0, d_{i}^{s}-z\right\}\right)-\mu z\right)
$$

Taking into account the ordering notations (27) the objective function $f_{i}(z)$ from (29) can be rewritten as follows:

$$
f_{i}(z)=\sum_{s=1}^{k(z)} \gamma_{[s]} \lambda_{i} a_{i} d_{i}^{[s]}+\sum_{s=k(z)+1}^{\tau} \gamma_{[s]} \lambda_{i} a_{i} z-\sum_{s=k(z)+1}^{\tau} \gamma_{[s]} \lambda_{i} b_{i}\left(d_{i}^{[s]}-z\right)-\mu z
$$

where

$$
k(z)=\max _{s}\left\{s: d_{i}^{[s]}<z\right\}
$$

After some algebraic manipulations this yields

$$
f_{i}(z)=\lambda_{i}\left(\sum_{s=1}^{k(z)} \gamma_{[s]}\left(a_{i}+b_{i}\right) d_{i}^{[s]}+\sum_{s=k(z)+1}^{\tau} \gamma_{[s]}\left(a_{i}+b_{i}\right) z\right)-\mu z-\lambda_{i} \sum_{s=t-\tau}^{t-1} \gamma_{s} b_{i} d_{i}^{s}
$$

This shows that $f_{i}(z)$ is piecewise linear function with the following slopes of the pieces:

$$
f_{i}^{\prime}(z)= \begin{cases}\lambda_{i}\left(a_{i}+b_{i}\right)-\mu & \text { if } z \leq d_{i}^{[1]} \\ \lambda_{i}\left(a_{i}+b_{i}\right) \sum_{s=k+1}^{\tau} \gamma_{[s]}-\mu & \text { if } d_{i}^{[k]} \leq z \leq d_{i}^{[k+1]}, k=1: \tau-1 \\ -\mu & \text { if } z \geq d_{i}^{[\tau]}\end{cases}
$$

The maximal value of this function is attained on the border between two pieces where the sign of the slope changes from positive to negative, or at zero if the first piece has negative slope. In the case when the slope of some piece is zero then all the points of the corresponding interval are the maximizers of $f_{i}(z)$. This yields the expression (28) for solution $V_{i}(\mu)=$ $z(\mu)$ of (29) which completes the proof.

The expression (28) can be used to compute the optimal allocation similarly to how (15)-(18) was used in the case of demand distributions with known density. Namely, it is necessary to select $\mu^{*}$ where the function

$$
\psi(\mu)=\sum_{i=1}^{m} V_{i}(\mu)
$$

crosses the level $\bar{V}$ and $V_{i}(\mu)$ is defined in (28). One should keep in mind that unlike in the case of distributions with density, the function $\psi(\mu)$ will be piecewise constant. Therefore it will be necessary to select a unique allocation from the set of possible candidates defined by $V\left(\mu^{*}\right)$ after $\mu^{*}$ will be found. 


\subsection{Threshold policy for controlling reallocation costs}

Let us summarize the moving window approach of the last section, extending it for the case when it is not desirable to have large allocation changes from one period to another.

Algorithm 5 Combined control and learning with moving window.

1. Initialization. At the beginning of some initial time period $t=0$ select the initial service resource allocation $V^{0}$. For example, this can be done by solving problem (12)-(13) with prior demand distributions. Use this allocation for service demand during period $t=0$.

2. Generic step. At the beginning of period $t$ we have observations of the past demand $d_{i}^{s}, s=0: t-1$ and the current resource allocation $V^{t-1}$. The following actions are performed at time $t$ :

2a. Decide the length of the time window $\tau_{s}$ and solve the problem (25), (13) with $\tau=\tau_{s}$. Its solution $\bar{V}^{t}$ will be the candidate for the new resource allocation.

2b. Allocate the service capacity $V^{t}$ during time period $t$ as a linear combination between the current allocation $V^{t-1}$ and the solution $\bar{V}^{t}$ :

$$
V^{t}=\left(1-\alpha_{t}\right) V^{t-1}+\alpha_{t} \bar{V}^{t}, \quad \alpha_{t} \in[0,1]
$$

The coefficient $\alpha_{t}$ serves the purpose of smoothing the allocation process and preventing drastic reallocation of resources between periods. The difference between solutions $\bar{V}^{t}$ and $\bar{V}^{t-1}$ of problem (25) for consecutive time windows can be considerable even though the difference between the corresponding optimal objective values may be small. For this reason taking these solutions as the current resource allocations may lead to considerable reallocation costs without bringing substantial benefits. Judicious selection of $\alpha_{t}$ is necessary in order to control this phenomenon. A simple way to make this selection is to choose $\alpha_{t}$ between 0 and 1 according to the following threshold policy.

Algorithm 6 Combined control and learning with moving window and threshold policy.

1. Initialization. Perform as in Algorithm 5 and additionally select the value of threshold $\Delta \geq 0$.

2. Generic step. Perform as in Algorithm 5 with the following modification to step $2 b$.

$2 b$. Let us denote by $F_{t}(V)$ the objective function in (25). Allocate the service capacity $V^{t}$ during time period $t$ as follows:

$$
V^{t}= \begin{cases}V^{t-1} & \text { if } F_{t}\left(\bar{V}^{t}\right)-F_{t}\left(\bar{V}^{t-1}\right)<\Delta \\ \bar{V}^{t} & \text { otherwise }\end{cases}
$$

The purpose of introducing this switching threshold is to control the reallocation costs. Let us show how the value $\Delta$ of the threshold can be selected from explicit consideration of such costs (8). At the beginning of time period $t$ the actual average performance during immediately preceding time horizon of length $\tau_{t}$ will be the following:

$$
\Psi_{t}(\Delta)=\frac{1}{\tau_{t}} \sum_{s=t-\tau_{t}}^{t-1} \sum_{i=1}^{m}\left(\lambda_{i}\left(a_{i} \min \left\{d_{i}^{s}, V_{i}^{s}\right\}-b_{i} \max \left\{0, d_{i}^{s}-V_{i}^{s}\right\}\right)-\delta_{i}\left|V_{i}^{s}-V_{i}^{s-1}\right|\right)
$$


Since the Algorithm 6 was used to generate the sequence of resource allocations $V^{s}$, this performance will depend on the value of threshold $\Delta$ used in this algorithm. Therefore the value of the threshold can be selected by fixing a sequence of times $t_{1}, t_{2}, \ldots, t_{k}, \ldots$ and optimizing the function $\Psi_{t}(\Delta)$ at these times. Suppose that $\Delta_{k}$ is the threshold value where $\Psi_{t_{k}}(\Delta)$ attains its maximum. Then the value of the threshold in (31) is kept constant and equal to $\Delta_{k}$ for time periods $t_{k}, \ldots, t_{k+1}-1$. The function $\Psi_{t}(\Delta)$ is quite complicated and its computation involves multiple solutions of problem (25). However, it depends only on a single parameter $\Delta$ and for this reason its optimization is quite feasible. In particular, given the computation times that are typical for these algorithms (see Sect. 6) it is feasible to apply the brute force approach by computing the values of this function on appropriate grid. Alternatively, one can consider Algorithm 5 with fixed value of $\alpha_{t}=\alpha_{k}$ for $t=t_{k}: t_{k+1}-1$. Instead of $\Delta$ the average performance in (32) will depend on $\alpha_{k}$. Then the optimal $\alpha_{k}$ can be obtain again by optimizing the average performance at time $t_{k}$.

In the context of stochastic optimization in finance, a similar form of online learning has been shown to satisfy certain asymptotic optimality properties (Gaivoronski and Stella 2003), and an application to index tracking in finance was presented in Gaivoronski et al. (2005). A separate investigation of the asymptotic properties of this procedure in the setting of this paper is one of the subjects of future research.

\subsection{Explicit treatment of risk}

So far we have looked at the resource allocations, which are optimal from the point of view of maximizing the integrated performance understood as the difference between revenue arising from servicing demand and costs arising from not meeting demand. However, it may be desirable to limit explicitly the risk of not servicing demand and select the service policy from the point of view of maximizing performance under explicit constraints on admissible risk. The first step in this approach is to define the relevant risk measure. There can be different such measures reflecting the different importance of sites, etc. In this section we shall consider as the measure of risk the total expected loss resulting from not meeting demand. Since it will be directly controlled, it will no longer be subtracted from the performance. The analogue of optimization problem (12)-(13) becomes the following:

$$
\begin{aligned}
& \max _{V_{i} \geq 0} \mathbb{E} \sum_{i=1}^{m} a_{i} \min \left\{d_{i}, V_{i}\right\} \\
& \mathbb{E} \sum_{i=1}^{m} b_{i} \max \left\{0, d_{i}-V_{i}\right\} \leq \sigma \\
& \sum_{i=1}^{m} V_{i} \leq \bar{V}
\end{aligned}
$$

Here $\sigma$ is the risk tolerance of the service provider that is explicitly fixed. The problem remains concave, therefore it is possible to employ duality theory in order to characterize the solutions of this problem. We report here the result obtained under the conditions of Corollary 3. More general results like those of Corollary 2 or Proposition 1 will have the similar structure.

Proposition 7 Suppose that the constraints (34), (35) are binding, that the distribution of demand has bounded marginal densities $h_{i}(y)$ with bounded supports that are connected sets and include 0. 
Then the solution of problem (33)-(35) exists, it is unique and has the form $V_{i}\left(\mu^{*}, \theta^{*}\right)$ where $\left(\mu^{*}, \theta^{*}\right)$ are obtained from solution of the system of two equations

$$
\begin{aligned}
& \sum_{i=1}^{m} V_{i}(\mu, \theta)=\bar{V} \\
& \sum_{i=1}^{m} b_{i} \int_{V_{i}(\mu, \theta)}^{\infty}\left(y-V_{i}(\mu, \theta)\right) h_{i}(y) d y=\sigma
\end{aligned}
$$

where

$$
V_{i}(\mu, \theta)= \begin{cases}z: \mathbb{P}\left(d_{i} \geq z\right)=\frac{\mu}{a_{i}+b_{i} \theta} & \text { if } 0 \leq \mu \leq a_{i}+b_{i} \theta \\ 0 & \text { if } \mu \geq a_{i}+b_{i} \theta\end{cases}
$$

Proof of this proposition is similar to the proof of Proposition 1 with the difference that here we have two Lagrangian multipliers. This makes the explicit treatment of risk somewhat more computationally demanding, although still well within the possibility of reasonably fast solution. Taking this result as a starting point it is possible to introduce reallocation costs, learning and threshold policies similarly to how it was done in Sects. 3.1-4.1.

\section{Risk budgeting}

In this section we want to model explicitly the situation where each service contract has a risk budget which is renewed periodically. Namely, for each site or set of sites corresponding to one customer there is an acceptable bound for the fraction of not serviced demand within a given time period. The adherence to this bound is reviewed periodically and there is a risk that steep penalties will be paid for failed adherence. For this reason the service resource allocation at a given period $t$ should take into account the current position with respect to these bounds. The service level agreements of this type can be found, for example, in the call center industry, in Robbins and Harrison (2010) they are called Horizon Based Service Level Agreements (HB-SLA). We refer the readers interested in details of such agreements and discussion of different types of contracts to this paper, Baron and Milner (2009), Milner and Olsen (2008) and extensive references there.

This situation can be modelled using the following notations:

$\Upsilon_{i}$ - the sequence of review times for site $i$ during some fixed time horizon $t=1: T$;

$\tau_{i}^{t-}$ - the last or current review time for site $i$;

$\tau_{i}^{t+}$ - the next review time for site $i$;

$D_{i}^{t}$ - the total service demand since the last review period for site $i$;

$D_{i+}^{t}$ - the total served demand since the last review period for site $i$;

$D_{i-}^{t}$ - the total not satisfied demand since the last review period for site $i$;

$R_{i}^{t}$ - the fraction of not satisfied demand since the last review period for site $i$.

More precisely,

$$
\begin{aligned}
& \tau_{i}^{t-}=\max _{\tau \leq t}\left\{\tau \mid \tau \in \Upsilon_{i}\right\}, \quad \tau_{i}^{t+}=\min _{\tau>t}\left\{\tau \mid \tau \in \Upsilon_{i}\right\} \\
& D_{i}^{t}=\sum_{s=\tau_{i}^{t-}}^{t-1} d_{i}^{s}, D_{i+}^{t}=\sum_{s=\tau_{i}^{t-}}^{t-1} \min \left\{d_{i}^{s}, V_{i}^{s}\right\}, \\
& D_{i-}^{t}=\sum_{s=\tau_{i}^{t-}}^{t-1} \max \left\{0, d_{i}^{s}-V_{i}^{s}\right\}, \quad R_{i}^{t}=\frac{D_{i-}^{t}}{D_{i}^{t}}
\end{aligned}
$$


The fraction $R_{i}^{t}$ of not satisfied demand should not exceed a target value $\zeta_{i}$ at the start of the next review period $\tau_{i}^{t+}$, otherwise the penalties proportional to the violation will be paid with the constant of proportionality $\lambda_{i}$. The total amount of these penalties will be

$$
f\left(V^{\tau}, d^{\tau}\right)=\sum_{i=1}^{m} \sum_{\tau_{i} \in \Upsilon_{i}} \lambda_{i} \max \left\{0, \frac{D_{i-}^{\tau_{i}}}{D_{i}^{\tau_{i}}}-\zeta_{i}\right\}
$$

where

$$
V^{\tau}=\left\{V_{i}^{t}\right\}_{i=1: m}^{t=1: T}, \quad d^{\tau}=\left\{d_{i}^{t}\right\}_{i=1: m}^{t=1: T}
$$

A more realistic penalty model here would be one which considers both fixed penalties for the mere fact of violation of the service contract and proportional penalties for the amount of violation. However, this will complicate the solution procedure and therefore will require much more computing time. For this reason we resort to approximation of reality which, however, retains a reasonable degree of adequacy. In practical situations, the violation of a contract may lead to fixed penalties, but it does not entail a penalty automatically. It is reasonable to assume for our modeling purposes that the larger the violation, the higher is the probability that contract violation will lead to a fixed penalty. Taking conditional expectation with respect to this probability distribution will bring the fixed penalty model closer to the proportional model.

The sequence of resource allocations $V^{\tau}$ should be chosen in order to minimize the expected penalties under resource constraints

$$
\begin{aligned}
& \min _{V^{\tau} \geq 0} \mathbb{E} f\left(V^{\tau}, d^{\tau}\right) \\
& \sum_{i=1}^{m} V_{i}^{t} \leq \bar{V}, \quad t=1: T
\end{aligned}
$$

where the expectation is taken with respect to the sequence of demands $d^{t}, t=1: T$ and $V_{i}^{t}$ is a function of demands observed up to time $t-1$. The problem (37)-(38) corresponds to the risk neutrality of the decision maker who manages the process. In the case of a risk averse decision maker, additional constraints on risk should be imposed that would reflect the risk preferences, similarly to Sect. 4.2. The first step in the formulation of the risk constraint is to introduce an appropriate risk measure. Usually it will be a measure of variability of performance, where in this case performance is measured by the function under expectation sign in (37). The traditional choice of risk measure is the variance of performance. In this case the risk constraint will take the form

$$
\operatorname{Var} f\left(V^{\tau}, d^{\tau}\right) \leq \sigma^{2}
$$

where $\sigma$ is defined by the risk tolerance of the decision maker. The problem to be solved in the case of explicit risk constraints will be then (37)-(39).

Exact solution of multiperiod dynamic problems (37)-(38) or (37)-(39) is far beyond computational feasibility. Therefore we resort to a stationary one period approximation.

Let us concentrate on the problem (37)-(38). The key concept which underlies the development is the current risk budget $q_{i}^{t}$ assigned to each site:

$$
q_{i}^{t}=\zeta_{i}-R_{i}^{t}
$$


This budget can be negative or positive. The one period stationary approximation will select the current resource allocation $V_{i}^{t}$ at the beginning of period $t$ in order to minimize the total expected negative part of the risk budget $Q_{-}^{t}$ by the end of the current period under the resource constraint:

$$
\begin{aligned}
Q_{-}^{t} & =\sum_{i=1}^{m} \lambda_{i} \mathbb{E}\left(\max \left\{0,-q_{i}^{t+1}\right\} \mid \mathbb{B}_{t}\right) \\
& =\sum_{i=1}^{m} \lambda_{i} \mathbb{E}\left(\max \left\{0, \frac{D_{i-}^{t}+\max \left\{0, d_{i}^{t}-V_{i}^{t}\right\}}{D_{i}^{t}+d_{i}^{t}}-\zeta_{i}\right\} \mid \mathbb{B}_{t}\right)
\end{aligned}
$$

The expectation here is taken conditioned on the history of the process described by a $\sigma$ field $\mathbb{B}_{t}$. In particular $D_{i-}^{t}$ and $D_{i}^{t}$ are measurable with respect to this field. Transforming (41) further we get:

$$
Q_{-}^{t}=\sum_{i=1}^{m} \lambda_{i} \mathbb{E}\left(\frac{1}{1+d_{i}^{t} / D_{i}^{t}} \max \left\{0, \frac{1}{D_{i}^{t}} \max \left\{0, d_{i}^{t}-V_{i}^{t}\right\}-\zeta_{i} \frac{d_{i}^{t}}{D_{i}^{t}}-q_{i}^{t}\right\} \mid \mathbb{B}_{t}\right)
$$

Let us now approximate this expression assuming that the unserved part of the demand is small compared with the served one and that the current period is sufficiently remote from the last review period for all sites. This is a reasonable assumption in the practical situations because the review period covers at least one month or longer and demand in the case of large organizations is seldom nonexistent. Then

$$
\frac{d_{i}^{t}}{D_{i}^{t}} \ll 1, \quad \frac{d_{i}^{t}}{D_{i}^{t}} \simeq \frac{\min \left\{d_{i}^{t}, V_{i}\right\}}{D_{i}^{t}}
$$

This allows to approximate (42) as follows:

$$
Q_{-}^{t} \simeq \sum_{i=1}^{m} \lambda_{i} \mathbb{E}\left(\max \left\{0, \frac{1}{D_{i}^{t}} \max \left\{0, d_{i}^{t}-V_{i}^{t}\right\}-\zeta_{i} \frac{\min \left\{d_{i}^{t}, V_{i}\right\}}{D_{i}^{t}}-q_{i}^{t}\right\} \mid \mathbb{B}_{t}\right)
$$

Even when these assumptions are not satisfied, (43) still gives a conservative estimate of (42). This yields the following optimization problem for determining the current resource allocation $V_{i}^{t}$ :

$$
\begin{gathered}
\min _{V_{i} \geq 0} \sum_{i=1}^{m} \frac{\lambda_{i}}{D_{i}^{t}} \mathbb{E}\left(\max \left\{0, \max \left\{0, d_{i}^{t}-V_{i}^{t}\right\}-\zeta_{i} \min \left\{d_{i}^{t}, V_{i}\right\}-q_{i}^{t} D_{i}^{t}\right\} \mid \mathbb{B}_{t}\right) \\
\sum_{i=1}^{m} V_{i} \leq \bar{V}
\end{gathered}
$$

This is a convex problem and it is possible to apply duality theory again in order to obtain the characterization of the optimal solutions. We do it here in the simplified (but the most practically important) setting of Corollary 3 in order to highlight the main features of the obtained solution. Full results similar to Proposition 1 can be obtained in a similar way.

Proposition 8 Suppose that the constraint (45), is binding and the distribution of demand has bounded marginal densities $h_{i}(y)$ with bounded supports that are connected sets and include 0 . 
Then the solution of problem (44)-(45) exists, it is unique and has the form $V_{i}\left(\mu^{*}\right)$ where $\mu^{*}$ is obtained by solving equation

$$
\sum_{i=1}^{m} V_{i}(\mu)=\bar{V}
$$

where

$$
V_{i}(\mu)=\left\{\begin{array}{lc}
0 & \text { if } \mu \geq \frac{\lambda_{i}}{D_{i}^{t}}\left(1+\zeta_{i}\right) \mathbb{P}\left\{d_{i}^{t} \geq q_{i}^{t} D_{i}^{t}\right\} \\
z^{*}: \mathbb{P}\left\{d_{i}^{t} \geq z^{*}\right\}=\mu \frac{D_{i}^{t}}{\lambda_{i}\left(1+\zeta_{i}\right)} & \text { if } \mu \leq \frac{\lambda_{i}}{D_{i}^{t}}\left(1+\zeta_{i}\right) \mathbb{P}\left\{d_{i}^{t} \geq q_{i}^{t} D_{i}^{t}\right\} \\
\bar{z}^{*}: \mathbb{P}\left\{d_{i}^{t} \geq\left(1+\zeta_{i}\right) \bar{z}^{*}+q_{i}^{t} D_{i}^{t}\right\}=\mu \frac{D_{i}^{t}}{\lambda_{i}\left(1+\zeta_{i}\right)} & z^{*} \leq-\frac{q_{i}^{t} D_{i}^{t}}{\zeta_{i}} \\
& \text { if } \mu \leq \frac{\lambda_{i}}{D_{i}^{t}}\left(1+\zeta_{i}\right) \mathbb{P}\left\{d_{i}^{t} \geq q_{i}^{t} D_{i}^{t}\right\} \\
z^{*} \geq-\frac{q_{i}^{t} D_{i}^{t}}{\zeta_{i}}
\end{array}\right.
$$

Proof Here we sketch the proof, referring to the detailed proof of Proposition 1.

First we prove the existence of solution through establishing the continuity of the objective function in (44) as it was done in the first part of the proof of Proposition 1. If we would consider here also the less practically important case when the resources are so abundant that the constraint (45) is not binding, then it would be necessary to consider the unconstrained problem (44) as the next step of the proof, similarly to Proposition 1 . Since we do not consider this case here, we go directly to the application of the duality theory, similarly to the step 3 of the proof of Proposition 1.

Firstly, we put the constraint (45) into Lagrangian with multiplier $\mu$. This has an effect that the minimization of this Lagrangian with respect to $V$ is decomposed into $m$ independent one dimensional problems of minimization with respect to $V_{i}$ with solutions dependent on $\mu$. Representing the expectation with integrals we obtain the following form for the objective function of these problems:

$$
\begin{gathered}
\bar{\psi}\left(\mu, V_{i}\right)=\left\{\begin{array}{l}
\frac{\lambda_{i}}{D_{i}^{t}} \int_{\left(1+\zeta_{i}\right) V_{i}+q_{i}^{t} D_{i}^{t}}^{\infty}\left(y-\left(1+\zeta_{i}\right) V_{i}-q_{i}^{t} D_{i}^{t}\right) h_{i}(y) d y+\mu V_{i} \quad \text { if } q_{i}^{t}>0 \\
\frac{\lambda_{i}}{D_{i}^{t}}\left(\mathbb{E} d_{i}^{t}-\left(1+\zeta_{i}\right) \int_{0}^{V_{i}^{t}} y h_{i}(y) d y\right. \\
\left.-\left(1+\zeta_{i}\right) V_{i} \int_{V_{i}^{t}}^{\infty} h_{i}(y) d y-q_{i}^{t} D_{i}^{t}\right)+\mu V_{i} \quad \text { if } q_{i}^{t} \leq 0, V_{i}^{t}<-\frac{q_{i}^{t} D_{i}^{t}}{\zeta_{i}} \\
\frac{\lambda_{i}}{D_{i}^{t}}\left(\int_{\left(1+\zeta_{i}\right) V_{i}+q_{i}^{t} D_{i}^{t}}^{\infty}\left(y-\left(1+\zeta_{i}\right) V_{i}-q_{i}^{t} D_{i}^{t}\right) h_{i}(y) d y\right. \\
\left.-\int_{0}^{-\frac{q_{i}^{t} D_{i}^{t}}{\zeta_{i}}}\left(\zeta_{i} y+q_{i}^{t} D_{i}^{t}\right) h_{i}(y) d y\right)+\mu V_{i} \quad \text { if } q_{i}^{t} \leq 0, V_{i}^{t} \geq-\frac{q_{i}^{t} D_{i}^{t}}{\zeta_{i}} \\
\mu-\frac{\lambda_{i}}{D_{i}^{t}}\left(1+\zeta_{i}\right) \mathbb{P}\left\{d_{i}^{t} \geq\left(1+\zeta_{i}\right) V_{i}+q_{i}^{t} D_{i}^{t}\right\} \quad \text { if } V_{i}^{t} \geq-\frac{q_{i}^{t} D_{i}^{t}}{\zeta_{i}}-\left(1+\zeta_{i}\right) V_{i} \mathbb{P}\left\{d_{i}^{t} \geq V_{i}\right\}
\end{array}\right.
\end{gathered}
$$

Differentiating this function with respect to $V_{i}$ we obtain:

$$
\frac{\partial \bar{\psi}\left(\mu, V_{i}\right)}{\partial V_{i}}= \begin{cases}\mu-\frac{\lambda_{i}}{D_{i}^{t}}\left(1+\zeta_{i}\right) \mathbb{P}\left\{d_{i}^{t} \geq V_{i}^{t}\right\} & \text { if } V_{i}^{t} \leq-\frac{q_{i}^{t} D_{i}^{t}}{\zeta_{i}} \\ \mu-\frac{\lambda_{i}}{D_{i}^{t}}\left(1+\zeta_{i}\right) \mathbb{P}\left\{d_{i}^{t} \geq\left(1+\zeta_{i}\right) V_{i}+q_{i}^{t} D_{i}^{t}\right\} & \text { otherwise }\end{cases}
$$


Having this representation of the derivative, we obtain the solution $V_{i}(\mu)$ for this onedimensional minimization problem by looking where the derivative changes the sign from minus to plus as we have done in Proposition 1. Since we have assumed that $h_{i}(y)$ has connected support that includes 0 this solution will be unique, in more general cases there will be sets where the derivative equals zero, as in Proposition 1. This study yields (46) and completes the proof.

Let us look now into some consequences of this result, considering the quantity

$$
D_{i q}^{t}=q_{i}^{t} D_{i}^{t}
$$

This is the cumulative amount of unserved demand during current review period which is violating the service target (if $q_{i}^{t}<0$ ) or current cumulative demand served in excess of service target (if $q_{i}^{t}>0$ ). Suppose that $\mu^{*}$ is the optimal value of the Lagrange multiplier. Then according to (46) the current optimal policy would be to allocate zero resources to a site $i$ in two cases, namely:

- The site has positive and large "unspent" risk budget $q_{i}^{t}, D_{i q}^{t}$ such that

$$
\mathbb{P}\left\{d_{i}^{t} \geq D_{i q}^{t}\right\} \leq \frac{\mu^{*}}{\lambda_{i} q_{i}^{t}\left(1+\zeta_{i}\right)} D_{i q}^{t}
$$

In other words, there is little value in adding additional resources since the SLA is very likely to be met in any case.

- The site has negative and large risk budget $q_{i}^{t}, D_{i q}^{t}$ such that allocation of service resources will not improve the situation considerably. This will happen when

$$
\left|D_{i q}^{t}\right| \geq \frac{\lambda_{i}\left|q_{i}^{t}\right|}{\mu^{*}}\left(1+\zeta_{i}\right)
$$

In other words, there is little value in adding additional resources since penalties are very likely to be incurred in any case.

\section{Numerical experiments}

In this section we report results of numerical experiments with the models described in the previous sections. We have described here several algorithms from the same family of lightweight stochastic optimization procedures that were tested numerically with hundreds of thousands of simulation runs. Besides changing a specific algorithm, we have changed in the course of simulations the number of sites (from 2 to 10000), demand distributions and other parameters of the problem. In this section we have included a representative selection of numerical results that show a typical behavior of our algorithms. We concentrate mostly on the risk budgeting approach of the Sect. 5, supplemented by methods from other sections, most notably the demand learning from Sect. 4. The reason for this selection is that we have not found substantial differences in the behavior of other algorithms and, besides, the risk budgeting model is closer to the specific industrial setting that has served as a motivation for this paper. Thus, we report here three series of experiments

1. Problems with known exact solution used for cross-evaluation.

2. Problems with unknown solution but known distribution of demand. 
3. Problems with unknown solution and unknown distribution of demand that is learned using arriving observations.

The experiments were performed with MATLAB, mostly on the HP EliteBook 8730w Mobile Workstation with Intel Core 2 Duo CPU running at $2.93 \mathrm{GHz}$ and $8 \mathrm{~GB}$ of memory, Windows 764 bit operating system, or on slightly inferior hardware.

\subsection{Cross-evaluation on problems with known solution}

The purpose of this section is to compare the service allocation policies from Sect. 5 with the best possible service allocation policies that utilize all the information available by the time period $t$ of the time horizon $1, \ldots, T$. Suppose that the only review of contracts occurs just after the completion of time period $T+1$. Then an optimal policy under the setting of Sect. 5 can be obtained by solving the problem

$$
\begin{gathered}
\min _{V^{1}} \mathbb{E}_{d^{1}} \min _{V^{2}} \mathbb{E}_{d^{2}} \cdots \min _{V^{T-1}} \mathbb{E}_{d^{T-1}} \min _{V^{T}} \mathbb{E}_{d^{T}} \sum_{i=1}^{m} \lambda_{i} \max \left\{0, \frac{D_{i-}^{T}}{D_{i}^{T}}-\zeta_{i}\right\} \\
\sum_{i=1}^{m} V_{i}^{t} \leq \bar{V}, \quad t=1: T
\end{gathered}
$$

Here the decision $V^{t}$ for $t>1$ depends on $\left(V^{\tau}, d^{\tau}\right)$ for $\tau=1: t-1$. This belongs to the domain of dynamic programming problems. We can write the state vector of this dynamic programming problem in terms of two components per site $i$. Those components are the total demand $D_{i}^{t}$ during the period $\tau=1: t$ and the total unserved demand $D_{i-}^{t}$ during this time. The state equations in this case will be as follows:

$$
\begin{aligned}
& D_{i}^{t+1}=D_{i}^{t}+d_{i}^{t} \\
& D_{i-}^{t+1}=D_{i-}^{t}+\max \left\{0, d_{i}^{t}-V_{i}^{t}\right\}
\end{aligned}
$$

Unfortunately, the solution of this problem is out of reach of the current computational capabilities except in the case of very small instances. We have tried to develop a test example that will be small enough to allow the exact solution, maintaining at the same time the main features of the problem. The simplest possible such example has two sites, $m=2$, with demands that assume values 0 and 1 with given probability. We have developed the MATLAB code that solves this problem exactly for $T=3$ by enumerating all possible combinations of demand and solving the resulting one dimensional optimization problems at each time period.

We have also developed a general MATLAB code for the stochastic dynamic programming (SDP) that implements the backward process for computing the expected future value function. At each time period starting from $t=T$ it performs the finite approximation of the state space by a multidimensional grid, the computation of the future expected value function at each point of the grid by solving the appropriate optimization problem and the interpolation of the future value function in order to solve the optimization problems at the previous time step. In this particular example the grid is four dimensional and the optimization problem has just one dimension. We have used MATLAB spline interpolation for the interpolation purposes. The aim of development of this code was to obtain the exact solutions for the example problem for the number of time periods that exceed 3 . The precision of SDP depends on how fine the grid is, so we have compared the solution obtained by 
Table 1 Comparison between the policy of Proposition 8 and the optimal policy

\begin{tabular}{lllll}
\hline$\Delta$ & $\operatorname{StDev}(\Delta)$ & $f^{*}$ & $\operatorname{StDev}\left(f^{*}\right)$ & $\delta$ \\
\hline 0.0956 & 0.0070 & 16.1747 & 0.3373 & $0.59 \%$ \\
\hline
\end{tabular}

SDP with the exact solution for $T=3$. Unfortunately, the results were very discouraging. For the grid that contained 5 points in each dimension (totally 625 states and about 40000 function interpolations necessary to perform one time step) the achieved best value of the objective function in (47) was inferior to the average value obtained by the application of the policy from Proposition 8 . For the grid that contained 11 points in each direction (14641 states and about 900000 interpolations at each time step) the result of SDP was very close to the exact optimal value, but it took about 19 hours of CPU time to obtain. For this reason we have abandoned the idea to use SDP in order to obtain the exact optimal values of the problem (47)-(48) for $T>3$ and have decided to perform our cross-evaluation with $T=3$.

The results of the comparison are very encouraging. The experiment was organized as follows.

1. The ranges for the values of the problem parameters were selected. There are five such ranges for each site:

$$
\begin{aligned}
& \lambda_{i} \in\left[\lambda_{i}^{\min }, \lambda_{i}^{\max }\right], \quad \zeta_{i} \in\left[\zeta_{i}^{\min }, \zeta_{i}^{\max }\right], \quad D_{i}^{0} \in\left[D_{i}^{0 \min }, D_{i}^{0 \max }\right] \\
& \gamma_{i}=\frac{D_{i-}^{0}}{D_{i}^{0}} \in\left[\gamma_{i}^{\min }, \gamma_{i}^{\max }\right], \quad p_{i} \in\left[p_{i}^{\min }, p_{i}^{\max }\right]
\end{aligned}
$$

where $p_{i}$ is the probability that demand at site $i$ equals 1 . The numerical values of these ranges and other parameters are given in the Appendix.

2. $K_{\text {problem }}$ of instances of the problem (47)-(48) were generated by sampling independently the parameter values from the uniform distributions on the ranges defined above. For each instance $k=1: K_{\text {problem }}$ we have performed $K_{\text {sim }}$ simulations of the policies from Proposition 8 and obtained the observations $f_{k i}$ of the objective function from (47), $i=1: K_{\text {sim }}$. These observations were averaged for each problem instance, which resulted in the average value $\hat{f}_{k}$ of the objective function (47) obtained by the policy from Proposition 8 .

3 . The exact optimal value $f_{k}^{*}$ of the instance $k$ of the problem (47)-(48) was computed. The difference $\Delta_{k}=\hat{f}_{k}-f_{k}^{*}$ gives the discrepancy between the value of the objective obtained by the policy from Proposition 8 and the best possible value. By averaging $\Delta_{k}$ the average discrepancy over the ranges of the problem parameters $\Delta$ was obtained. The average $f^{*}$ of the optimal values gives the average optimal value of (47)-(48) over the parameter ranges. Finally, the ratio $\delta=100 \% \times \Delta_{k} / f^{*}$ gives the average relative discrepancy between the values obtained by our policy and the best possible policy. The results of this experiment together with the relevant standard deviations are reported in Table 1.

Thus, the policy of Proposition 8 is worse than the best possible policy only by a few tenths of one percent. We consider this very good performance for this example.

\subsection{The case of known demand distribution: service dimensioning curves}

The purpose of this series of experiments was to shed light on the behavior of our service allocation policies for more realistic systems consisting of up to one thousand service sites in the case when the decision maker knows the distribution of demand. A more realistic time horizon of $T=30$ was taken for these experiments. The selection of results presented here shows how the average risk budgets change with the change of the total service capacity, the 
number of service sites and the volatility of demand. Such experiments can help the decision maker to dimension the service resources according to her preferences.

Experiment 1 This experiment shows how the average risk budget obtained by the review time depends on the number of service sites and the volatility of demand. It was organized as follows.

1. The total average service demand $\bar{D}$ is selected that is generated by the varying number $m$ of homogeneous sites. They generate the demand independently according to the same known distribution. We report here the results for the uniform distribution, similar results we have obtained for the exponential distribution. Thus, in this case each of $m$ sites generates the demand $d$ distributed uniformly on the interval $[0,2 \bar{D} / \mathrm{m}]$. The volatility of demand is measured by its standard deviation that in this case will be

$$
\operatorname{StDev}(d)=\frac{\bar{D}}{m \sqrt{3}}
$$

Thus, for fixed $m$ the demand volatility is proportional to the total average demand $\bar{D}$ such that $k$ times larger demand will have $k$ times larger volatility. For this reason we call the parameter $k$ relative demand volatility. Another important parameter that was selected is the ratio $\rho$ between the average service demand and the total service capacity $\bar{V}$, we call it the capacity/demand ratio:

$$
\bar{V}=\rho \bar{D}
$$

2. We fixed $\bar{D}$ and selected the problem parameters $\lambda_{i}, \zeta_{i}, D_{i}^{0}$ and $\gamma_{i}$ to be the same for all sites. After that we performed simulations of the policy in Proposition 8 policy for different values of $m, k$ and $\rho$ during the time horizon $T=30$. For each selection $(m, k, \rho)$ we have performed $K_{\text {sim }}$ simulations and after each simulation we have obtained the value of the risk budget for site $i$

$$
q_{i}^{T}=\zeta_{i}-\frac{D_{i-}^{T}}{D_{i}^{T}}
$$

by the review time. Since all sites are chosen to be homogeneous, we have averaged the obtained risk budgets between sites. After that we have obtained the mean final risk budget by averaging with respect to $K_{\text {sim }}$ simulations and computed the standard deviation of obtained estimate. The results are shown in Figs. 1-4. The vertical axis on these and other figures show the average risk budget by the time of review, the desirable values being nonnegative or at least close to zero.

Figures 1 and 2 show the dependence of the average final risk budget on the number of sites that share the same value of average demand. One can see that the larger the number of sites, the closer the risk budget is to the desired values. When the number of sites is several hundred it is indeed very close to zero. For small number of sites its value can be considerably lower than desired. This is due to our choice of the amount of the service capacity that on these figures is equal to the average total demand. This confirms the phenomenon well known in other similar cases: for reliable service the service capacity should exceed the average demand by a certain amount that depends on the volatility of demand. We shall return to this issue in Experiment 2. These figures also show that the higher the demand volatility, the further the risk budget is from the target, and the slower its convergence to zero as the number of sites increases. These curves can be used by the decision maker to dimension the 
Fig. 1 Dependence of the average final risk budget on the fixed service capacity and different relative demand volatilities and with capacity/demand ratio, $\rho=1$. The maximal standard deviation of the points on the curves is 0.0024 numbers of service sites for the

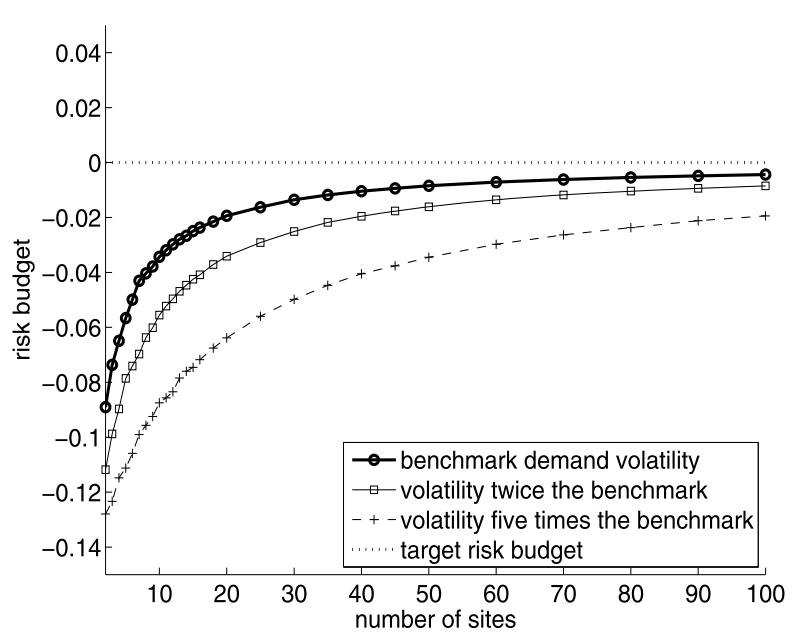

Fig. 2 Dependence of the average final risk budget on the numbers of service sites for the fixed service capacity and different relative demand volatilities and with capacity/demand ratio, $\rho=1$, larger number of sites. The maximal standard deviation of the points on the curves is 0.0024

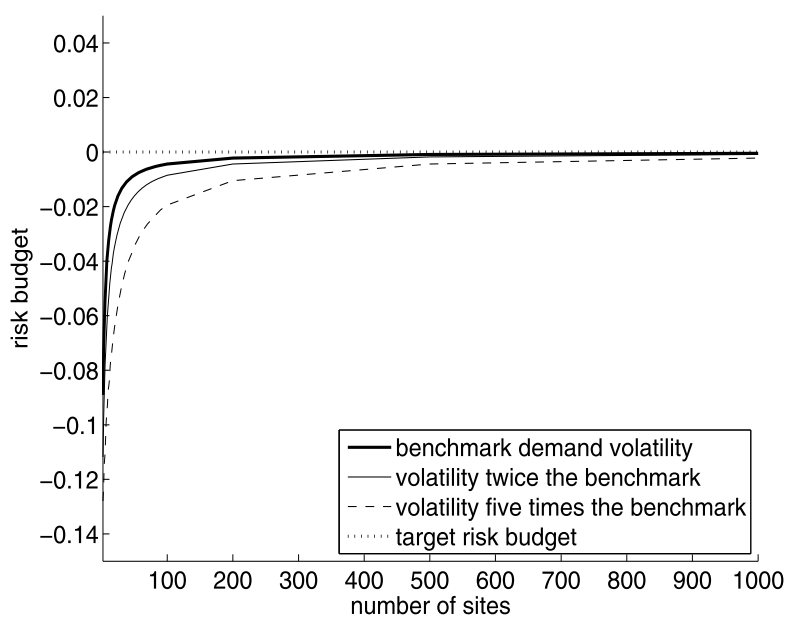

service center by striking a balance between the number of sites attached to a service center, attainable risk budget and the amount of resources allocated to a service center.

Figures 3 and 4 provide a complementary view showing how the average final risk budget depends on the relative volatility of demand. One can notice that at first the risk budget deteriorates quickly with the increase in volatility, but then it stabilizes and there is little difference in the average risk budget as the relative demand volatility varies from 20 to 100 . These stabilization values are considerably below the desired ones if the service capacity is equal to the average demand, as Fig. 3 suggests. However, the Fig. 4 shows that the behavior of the risk budget is much closer to the desired values if the service capacity is increased by $25 \%$. Both figures confirm that the effects of the volatility increase are somewhat mitigated if the same amount of the demand is generated by a larger number of sites. These figures complement Figs. 1, 4 for the purpose of dimensioning by a decision maker.

Experiment 2 This experiment shows how one can strike a balance between the attainable risk budget and other important characteristics of the system, like the total allocated service 
Fig. 3 Dependence of the average final risk budget on the relative volatility of demand for the fixed service capacity and different numbers of sites, capacity/demand ratio $\rho=1$. The maximal standard deviation of the points on the curves is 0.00083

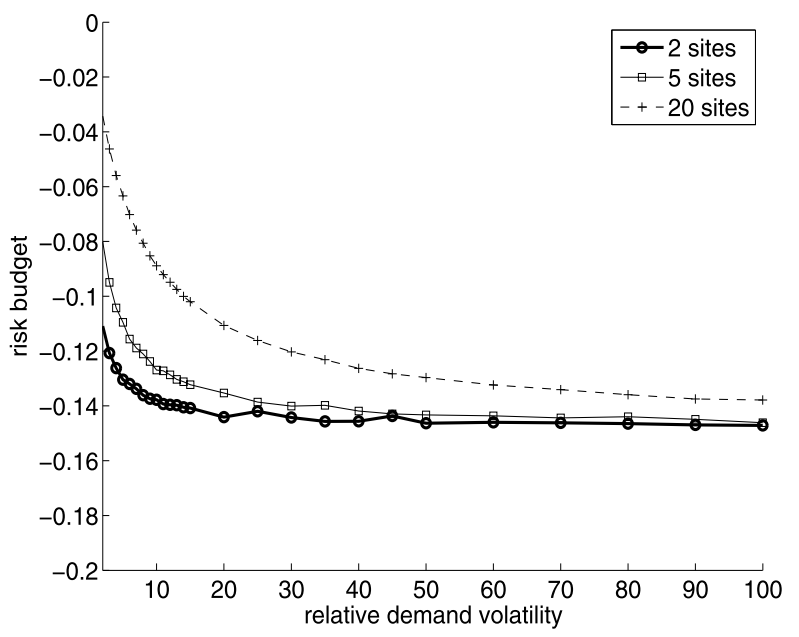

Fig. 4 Dependence of the average final risk budget on the relative volatility of demand for the fixed service capacity and different numbers of sites, capacity/demand ratio $\rho=1.25$. The maximal standard deviation of the points on the curves is 0.00066

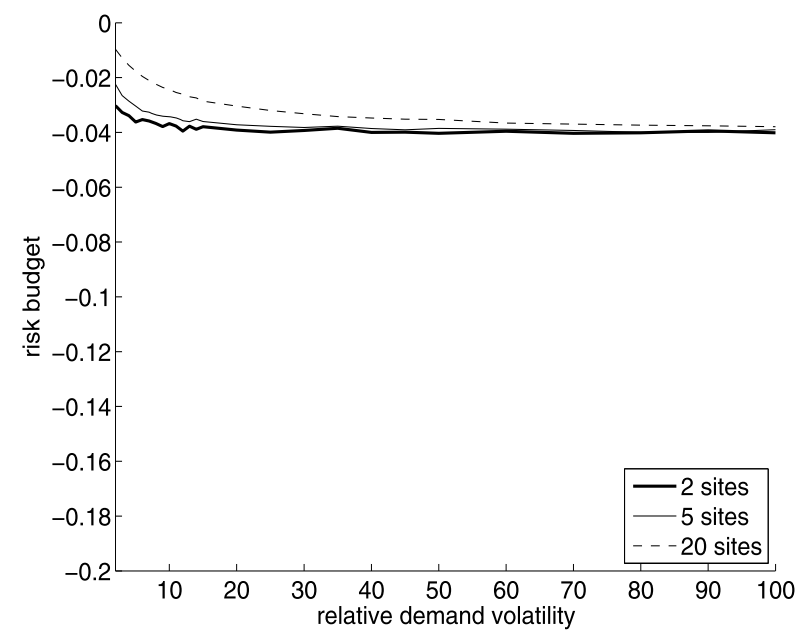

capacity and the idle time. It was organized like Experiment 1 except that we have considered the system consisting of four demand generating sites with different demand volatility. The demand was distributed uniformly for all sites starting from zero and up to an upper bound that was different for different sites and took the values $\bar{d}, 2 \bar{d}, 4 \bar{d}$ and $8 \bar{d}$ correspondingly for some fixed $\bar{d}$. Thus, the total average demand $\bar{D}$ was $7.5 \bar{d}$ for this system. The service capacity $\bar{V}$ was chosen according to (49). After choosing the problem parameters $\lambda_{i}, \zeta_{i}, D_{i}^{0}, \gamma_{i}$ and $p_{i}$ we have performed $K_{\text {sim }}$ simulations for each of the chosen values of the capacity/demand ratio $\rho$ and computed the average values of the final risk budget and its standard deviation, as in Experiment 1. The results of this experiment are shown on Figs. 5, 6.

Figure 5 shows the dependence of the average final risk budgets on the capacity/demand ratio $\rho$ for all four sites. One can notice that if demand overpowers the service capacity then the performance is understandably dismal. But the interesting thing is the different treatment reserved for different sites. The site with the largest demand variation is practically 
Fig. 5 Dependence of the average final risk budget on the ratio $\rho$ between the service capacity and the total average demand for sites with different volatilities and average demands. The maximal standard deviation of the points on the curves is 0.0024

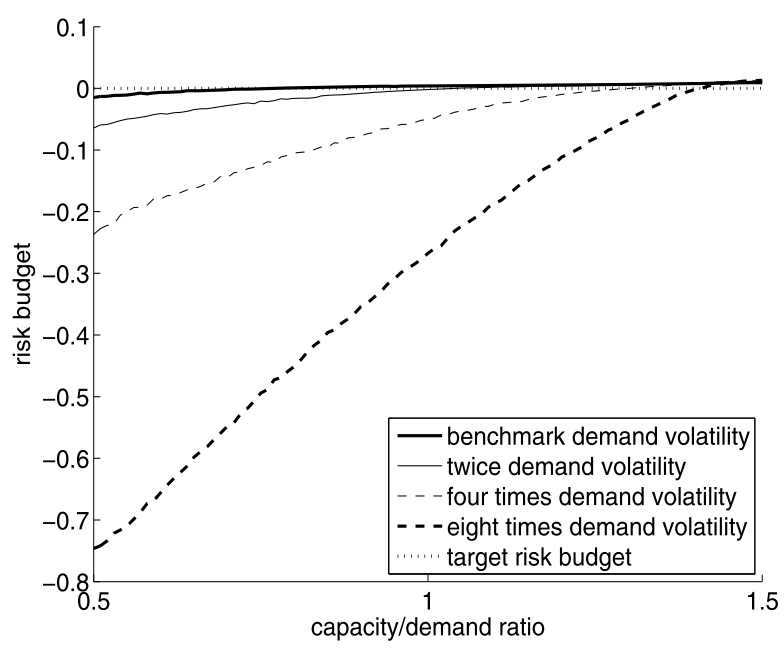

Fig. 6 Dependence of the average final risk budget on the fraction of idle time (unused allocated service capacity). The maximal standard deviation of the points on the curve is 0.0025 vertically and 0.024 horizontally

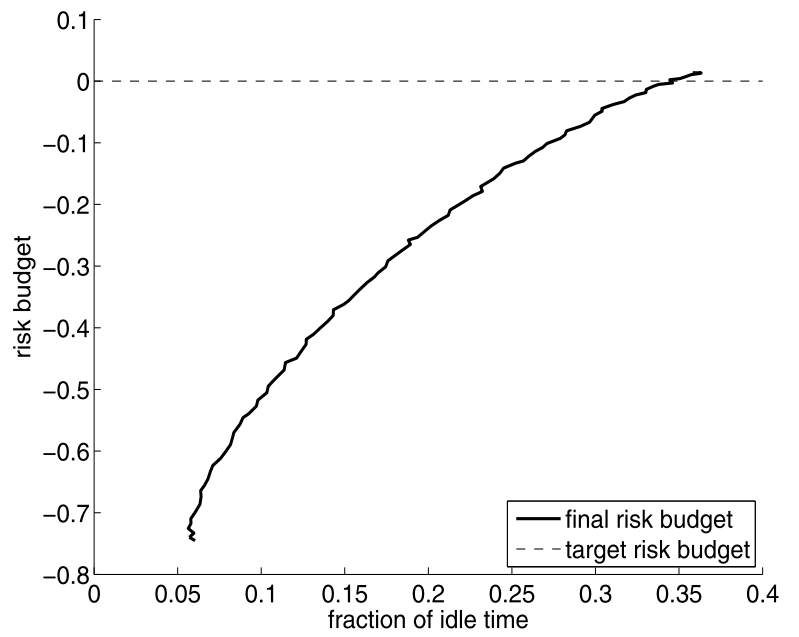

abandoned because the system does have a chance to serve it properly (with $\rho=0.5$ even the whole capacity is not enough to satisfy all the demand from the largest site). Instead, the system concentrates on achievable goals, that is the satisfaction of demand from the first two less volatile and smaller sites. Indeed, the risk budgets for these sites are acceptable even with half of the total resource coverage. One should add that all the sites were considered equally important in this example by selecting the same values of the weights $\lambda_{i}$. This behavior can be modified substantially by choosing unequal $\lambda_{i}$ as we shall see in the next subsection. With more resources added, the performance improves markedly. It becomes very good for the first two sites when the capacity becomes equal to the total average demand, the same happens with the second largest site when the capacity exceeds the demand by $25 \%$ and finally the performance for the largest site becomes excellent when the capacity excess reaches $40 \%$. Similar figures can help the decision maker to strike a balance between the performance and total allocated capacity. 
Table 2 Dependence of computing time in seconds on the number of sites

\begin{tabular}{lllll}
\hline Number of sites & 10 & 100 & 1000 & 10000 \\
\hline Average cpu time & 0.0738 & 0.6432 & 6.443 & 65.55 \\
Standard deviation & 0.0016 & 0.0082 & 0.023 & 0.15 \\
\hline
\end{tabular}

The final in this section Fig. 6 shows another aspect of capacity/demand trade-off. It presents the dependence between the idle service time and the resulting risk budget. The fraction of the idle time shown on the horizontal axis is computed for each site as the ratio between the allocated but not used service resources and the total allocated resources. As one can expect, the greater the fraction of idle time, the higher the final risk budget. If about one third of resources goes overallocated then the performance is excellent. It is interesting that this curve is the same for all sites within statistical errors.

Experiment 3 The purpose of this experiment was to see how the computing time depends on the number of sites. We measured the computing time that MATLAB code consumed during 30 time steps of simulation for different number $K_{\text {sites }}$ of sites. The average computing time among $K_{\text {sim }}$ randomly generated instances was computed. The problem parameters were selected according to uniform distributions as in Sect. 6.1, the demand was distributed uniformly between $\left[0, d_{\max }\right]$ where $d_{\max }$ was selected randomly for each site from the uniform distribution on $\left[d_{\max }^{-}, d_{\max }^{+}\right]$. The results are reported in the Table 2 and show the linear growth in computing time.

Summarizing, the experiments of this section highlight the important features of the policy of Proposition 8 and show how the system can be dimensioned taking into account different and conflicting requirements. The numerical values of different parameters used in these experiments are reported in the Appendix.

\subsection{The case of demand learning}

In this section we describe an experiment where the control algorithm does not know the exact demand distribution and learns demand with the arrival of its observations as in Sect. 4. Thus, the algorithm combines the settings of Sects. 4 and 5.

In order to access the performance of this resource allocation algorithm a simulation model of the controlled system was developed in MATLAB. It consisted of the following components.

1. Model of the controlled system which generates service demand. Every simulation step it generates the demand streams for each site according to some demand arrival distribution specific for each site. It also generates the service times required by each demand which are the observations from some probabilistic distributions. In this way each site at the end of each period generates the service demand expressed in terms of service time.

2. Model of control system which is responsible for the service resource allocation. At the beginning of each period it performs the following actions.

- Learning of the demand distribution. This is done on the basis of the demands observed during a specified number of previous periods.

- Service resource allocation. This is done by solving problem (44)-(45) where the estimate of the probability distribution of demand obtained during the learning stage is used instead of actual demand distribution which remains unknown to the control system. The 
expression (46) from Proposition 8 is used for this purpose together with MATLAB function $f$ zero for computing the optimal value of the Lagrange multiplier.

- Performance evaluation. This is done at the end of each period when the actual service demand for this period becomes known to the control system and it is possible to see how the actual demand differs from the service resource allocation done at the beginning of the period. The current risk budgets $q_{i}$ from (40) which will be used at the beginning of the next period in the resource allocation problem (44)-(45) are computed at this stage.

In what follows we report one example of performance of this system that illustrates the typical behavior of the algorithm. The controlled system is composed of 5 sites. Each site generates the demand arriving according to the Poisson distribution with the service times being distributed exponentially. The numerical values of the distribution parameters are shown in the Appendix, Table 3.

Figures 7-11 show the results of the experiments. In the previous section we have shown the average behavior at the end of control horizon. Here we complement the picture by presenting the typical behavior of the current risk budgets in the course of simulations.

As in the previous sections, the vertical axis on all these figures shows the value of the risk budgets $q_{i}$ for different sites. The service target $\zeta_{i}$ for the fraction of unserved demand was the same for all sites and equal to 0.1 . For this reason the possible range of the risk budgets is between -0.9 and 0.1 with zero meaning that the performance is right on target. The risk budgets are measured as the difference between the target for the fraction of unserved demand and the actual unserved demand during the fixed number of time periods. This number of periods was 250 for Figs. 7-10 and 30 for Fig. 11. Everywhere except in Fig. 8 the site weights $\lambda_{i}$ were equal. The total simulation horizon consisted of 1000 periods.

Figures 7-11 show the time dependence of the risk budgets and the horizontal axis on these figures shows the number of periods. Figure 7 shows what happens when the total amount of service resources is not enough to meet all the service demand. More specifically, the total amount of service resource $\bar{V}$ is 0.75 of the total average demand in each period. One can see that indeed, most of the sites are off the service target as one could expect. However the two sites 2 and 4 with the smallest expected demand and the least demand variation are not far from meeting the target. One can also observe the transient period of the

Fig. 7 Time dependence of risk budgets. 250 day review period, 0.75 service resource coverage, equal importance of sites. There is one curve for each of the five sites

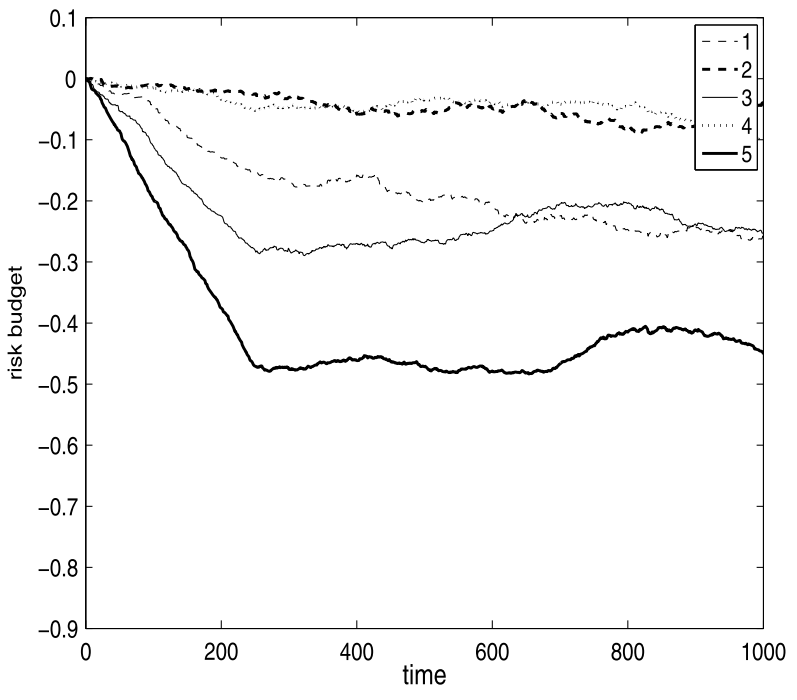


Fig. 8 Time dependence of risk budgets. 250 day review period, 0.75 service resource coverage, site 5 is of prime importance. There is one curve for each of the five sites

Fig. 9 Time dependence of risk budgets. 250 day review period, 1.00 service resource coverage, equal importance of sites. There is one curve for each of the five sites
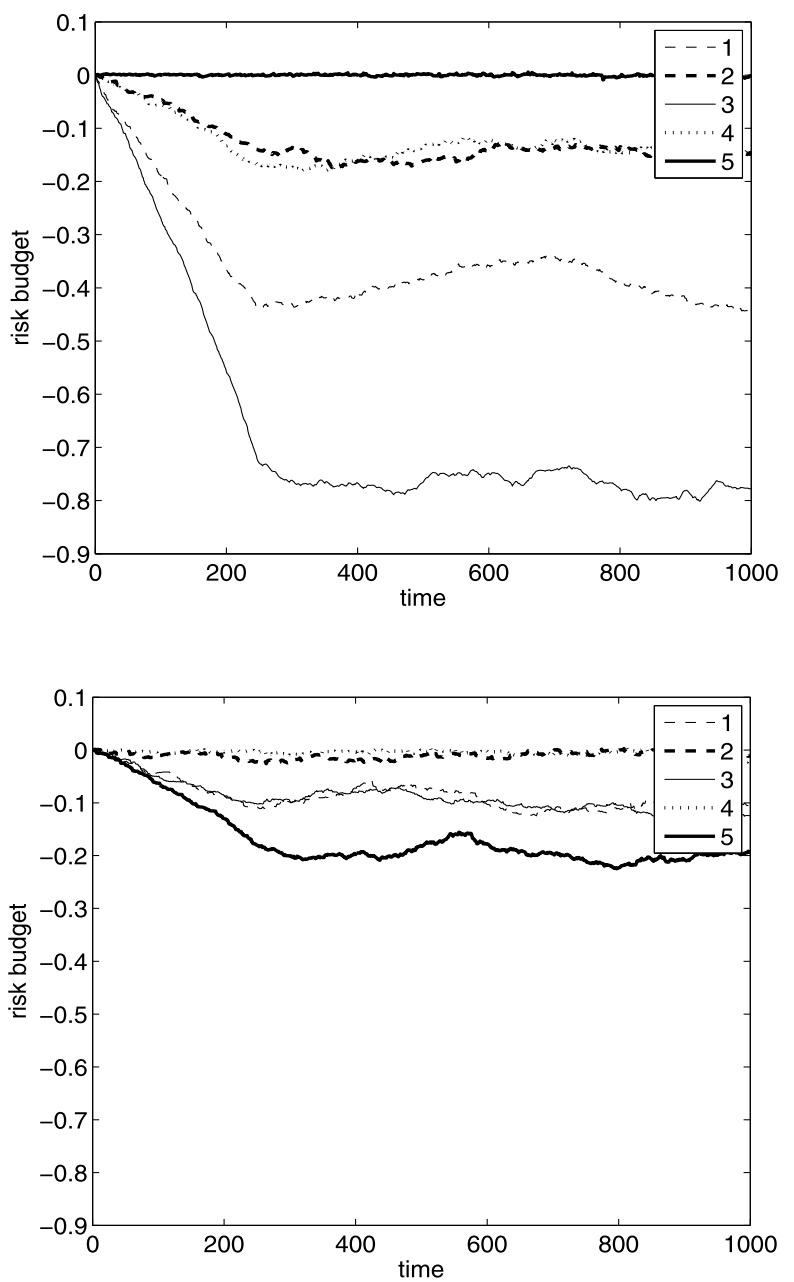

first 250 days when the risk budgets drifted from the initial value of zero to their respective values during the last 750 days with oscillations around these values.

What happens when the sites differ in their importance and the prime quality of service must be delivered to certain sites even when the total resources are lacking? Figure 8 answers this question. There it is assumed that the site 5 which is the worst served site on Fig. 7 should instead be offered prime quality service. This is achieved by assigning the site importance weight $\lambda_{5}$ to be 10 times higher than for the rest of the sites. One can see from Fig. 8 that the quality of service for site 5 has become excellent indeed, but the QoS for other sites has deteriorated, which could be expected given the overall lack of service resources.

Figures 9 and 10 show that the QoS increases with an increase in the total available service resources $\bar{V}$. This amount is equal to the total average demand in Fig. 9. Of course, this is still not sufficient for the target QoS given the demand variability. However, Fig. 9 shows that performance has increased substantially and for the sites 2 and 4 with the smallest expected demand it is right on the QoS target. The site importance weights $\lambda_{i}$ starting from this figure are again all equal. 
Fig. 10 Time dependence of risk budgets. 250 day review period, 1.25 service resource coverage, equal importance of sites. There is one curve for each of the five sites

Fig. 11 Time dependence of risk budgets. 30 day review period, 1.25 service resource coverage
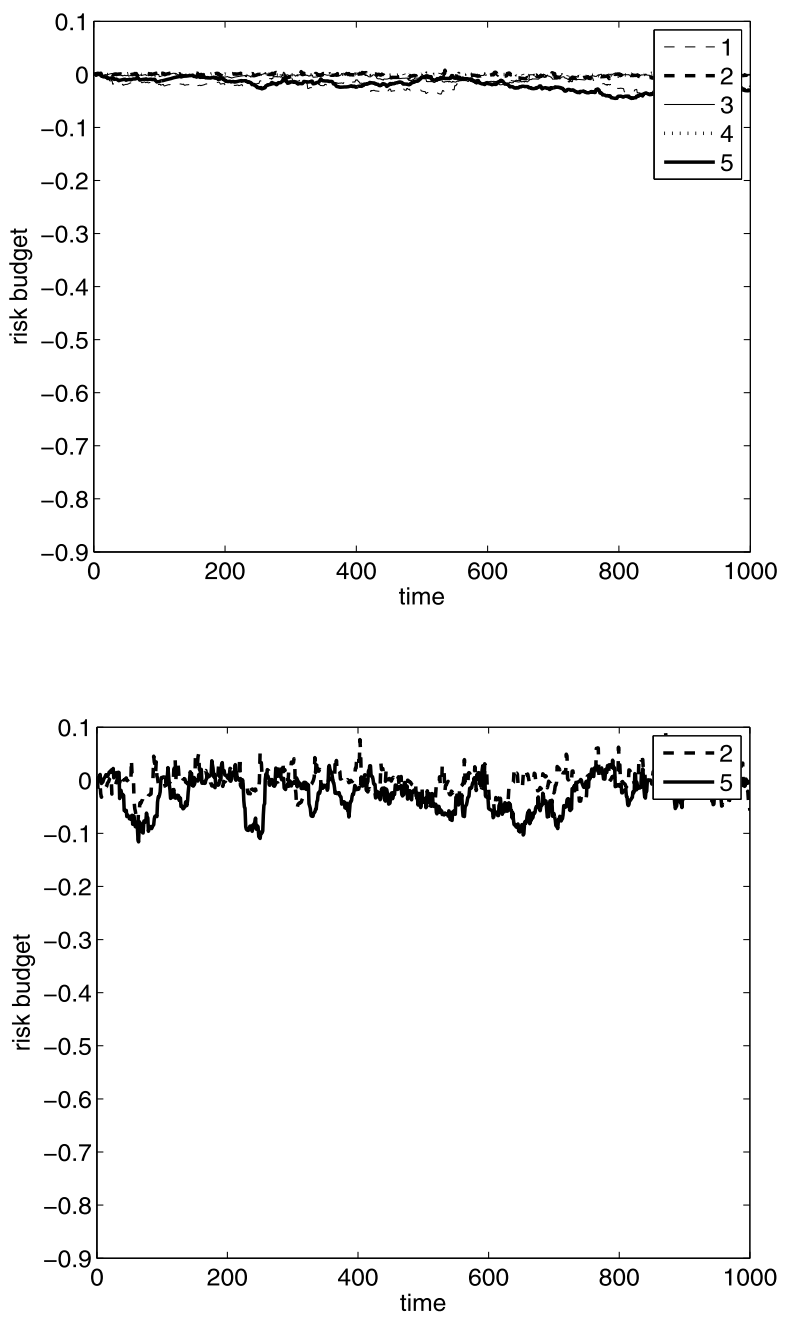

When the amount of service resources $\bar{V}$ increases even further to the level of 1.25 of the average demand then Fig. 10 shows that the QoS target is met practically for all sites with slight deviation only for the sites with the largest demand. This conforms with the findings of the previous section.

Figure 11 shows what happens when the review period for the performance measurement is reduced from 250 days to 30 days, all the rest being equal to the conditions of Fig. 10. As expected, the value of the performance measure now has a considerably larger variance, being in the band around the QoS target. Two sites are shown: the site 2 with the smallest demand and the site 5 with the largest demand. If the performance variability is judged to be too high, it can be improved by raising the resource level.

Our experiment show that the learning process allows to reach the performance targets in terms of the risk budget also in the absence of precise information about the distribution of demand. 
Table 3 Parameters of demand distributions for each site

\begin{tabular}{llllll}
\hline site & 1 & 2 & 3 & 4 & 5 \\
average number of failures & 5 & 7 & 10 & 15 & 11 \\
average service times & 10 & 5 & 7 & 3 & 8 \\
expected demand & 50 & 35 & 70 & 45 & 88 \\
\hline
\end{tabular}

\section{Conclusions}

This paper shows that a combination of online stochastic optimization with learning can lead to effective resource allocation algorithms under considerable demand uncertainty and varying performance targets. This approach is not limited to resource allocation among competing sites, but can be applied to many other problems in service and production control and supply chain management.

Acknowledgements The authors are grateful to two anonymous reviewers who provided numerous insightful comments that have helped to improve the paper considerably.

Open Access This article is distributed under the terms of the Creative Commons Attribution Noncommercial License which permits any noncommercial use, distribution, and reproduction in any medium, provided the original author(s) and source are credited.

\section{Appendix}

1. The values of parameters for the experiment of Sect. 6.1 .

The parameters for the both sites were drawn from the same ranges: $\lambda_{i}^{\min }=50, \lambda_{i}^{\max }=$ $150, \zeta_{i}^{\min }=0.05, \zeta_{i}^{\max }=0.2, D_{i}^{0 \min }=1, D_{i}^{0 \max }=20, \gamma_{i}^{\min }=0.05, \gamma_{i}^{\max }=0.3$, $p_{i}^{\min }=0.25, p_{i}^{\max }=0.75, K_{\text {problem }}=1000, K_{\text {sim }}=10000$.

2. The values of parameters for the experiments of Sect. 6.2.

Experiment $1: \lambda_{i}=100, \zeta_{i}=0.1, D_{i}^{0}=10, \gamma_{i}=0.1, \bar{D}=1$.

Figures 1, 2: $K_{\text {sim }}=100$, the total number of simulations: 9300 , cpu time: $4815.1 \mathrm{sec}-$ onds.

Figures 3, 4: $K_{\text {sim }}=1000$, the total number of simulations: 162000 , cpu time 10482 seconds.

Experiment 2: $\lambda_{i}=100, \zeta_{i}=0.1, D_{i}^{0}=10, \gamma_{i}=0.1, \bar{d}=1$.

Figure 5: $K_{\text {sim }}=1000$, the total number of simulations: 101000 , cpu time 3696 seconds.

Figure 6: $K_{\text {sim }}=1000$, the total number of simulations: 101000 , cpu time 3806 seconds.

Experiment 3: $K_{\text {sim }}=10, \lambda_{i}^{\min }=50, \lambda_{i}^{\max }=150, \zeta_{i}^{\min }=0.05, \zeta_{i}^{\max }=0.2, D_{i}^{0 \min }=1$, $D_{i}^{0 \max }=20, \gamma_{i}^{\min }=0.05, \gamma_{i}^{\max }=0.3, d_{\max }^{-}=1, d_{\max }^{+}=10$.

3. The values of parameters for the experiments of Sect. 6.2 .

\section{References}

Abdel-Malek, L. L., \& Montanari, R. (2005). An analysis of the multi-product newsboy problem with a budget constraint. International Journal of Production Economics, 97, 296-307.

Aksin, Z., Armony, M., \& Mehrotra, V. (2007). The modern call center: A multi-disciplinary perspective on operations management research. Production and Operations Management, 16(6), 665-688. 
Atlason, J., Epelman, M. A., \& Henderson, S. G. (2008). Optimizing call center staffing using simulation and analytic center cutting-plane methods. Management Science, 54(2), 295-309.

Auer, P., Cesa-Bianchi, N., \& Gentile, C. (2002). Adaptive and self-confident on-line learning algorithms. Journal of Computer and System Sciences, 64(1), 48-75.

Avramidis, A. N., Chan, W., Gendreau, M., L'Ecuyer, P., \& Pisacane, O. (2010). Optimizing daily agent scheduling in a multiskill call center. European Journal of Operational Research, 200, 822-832.

Baron, O., \& Milner, J. (2009). Staffing to maximize profit for call centers with alternate service-level agreements. Operations Research, 57(3), 685-700.

Bertsekas, D. P. (2007). Dynamic programming and optimal control (3d ed.). Nashua: Athena Scientific.

Birge, J. R., \& Louveaux, F. (1997). Introduction to stochastic programming. New York: Springer.

Cesa-Bianchi, N., \& Lugosi, G. (2006). Prediction, learning, and games. Cambridge: Cambridge University Press.

Chung, C.-S., Flynn, J., \& Kirca, O. (2008). A multi-item newsvendor problem with preseason production and capacitated reactive production. European Journal of Operational Research, 188, 775-792.

Cover, T. M. (1991). Universal portfolios. Mathematical Finance, 1(1), 1-29.

de Farias, D. P., \& Van Roy, B. (2006). A cost-shaping linear program for average-cost approximate dynamic programming with performance guarantees. Mathematics of Operations Research, 31(3), 597-620.

Dempster, M. A. H., Lenstra, J. K., \& Rinnooy Kan, A. H. G. (Eds.) (1982). Deterministic and stochastic scheduling. Reidel: Dordrecht.

Erlebacher, S. J. (2000). Optimal and heuristic solutions for the multi-item newsvendor problem with a single capacity constraint. Production and Operations Management, 9, 303-318.

Gaivoronski, A. A. (2005). SQG: Stochastic programming software environment. In S. W. Wallace \& W. T. Ziemba (Eds.), Applications of stochastic programming. Philadelphia: SIAM \& MPS.

Gaivoronski, A. A., \& Stella, F. (2003). On-line portfolio selection using stochastic programming. Journal of Economic Dynamics \& Control, 27, 1013-1043.

Gaivoronski, A. A., Krylov, S., \& Van der Wijst, D. (2005). Optimal portfolio selection and dynamic benchmark tracking. European Journal of Operational Research, 163.

Hadley, G., \& Whitin, T. (1963). Analysis of inventory systems. Englewood Cliffs: Prentice-Hall.

Harrison, J. M., \& Zeevi, A. (2005). A method for staffing large call centers based on stochastic fluid models. Manufacturing \& Service Operations Management, 7(1), 20-36.

Kalai, A., \& Vempala, S. (2005). Efficient algorithms for on-line optimization. Journal of Computer and System Sciences, 71(3), 266-290.

Kall, P., \& Wallace, S. (1994). Stochastic programming. New York: Wiley.

Konda, V. R., \& Tsitsiklis, J. N. (2003). On actor-critic algorithms. SIAM Journal on Control and Optimization, 42(4), 1143-1166.

Lau, H.-S., \& Lau, A. H.-L. (1996). The newsstand problem: A capacitated multiple-product single-period inventory problem. European Journal of Operational Research, 94, $29-42$.

Megow, N., \& Schulz, A. S. (2004). On-line scheduling to minimize average completion time revisited. Operations Research Letters, 32, 485-490.

Milner, J. M., \& Olsen, T. L. (2008). Service-level agreements in call centers: Perils and prescriptions. Management Science, 54(2), 238-252.

Patriksson, M. (2008). A survey on the continuous nonlinear resource allocation problem. European Journal of Operational Research, 185, 1-46.

Pereira, M. V. F., \& Pinto, L. M. V. G. (1991). Multi-stage stochastic optimization applied to energy planning. Mathematical Programming, 52, 359-375.

Pflug, G. Ch. (1996). Optimization of stochastic models. The interface between simulation and optimization. Boston: Kluwer.

Philpott, A. B., \& Guan, Z. (2008). On the convergence of stochastic dual dynamic programming and related methods. Operations Research Letters, 36, 450-455.

Powell, W. B. (2009). What you should know about approximate dynamic programming. Naval Research Logistics, 56, 239-249.

Powell, W. B., Marar, A., Gelfand, J., \& Bowers, S. (2002). Implementing real-time optimization models: A case application from the motor carrier industry. Operations Research, 50(4), 571-581.

Robbins, T. R., \& Harrison, T. P. (2010). A stochastic programming model for scheduling call centers with global service level agreements. European Journal of Operational Research, 207, 1608-1619.

Ruszczynski, A., \& Shapiro, A. (Eds.) (2003). Stochastic programming, handbook in operations research and management science. Amsterdam: Elsevier Science.

Van Hentenryck, P., Bent, R., Mercier, L., \& Vergados, Y. (2009). Online stochastic reservation systems. Annals of Operation Research, 171(1), 101-126.

Zhang, B., \& Du, S. (2010). Multi-product newsboy problem with limited capacity and outsourcing. European Journal of Operational Research, 202, 107-113.

Zhang, B., Xu, X., \& Hua, Z. (2009). A binary solution method for the multi-product newsboy problem with budget constraint. International Journal of Production Economics, 136-141, 117. 